\title{
MULTI-SCALED DIFFUSION-APPROXIMATION. APPLICATIONS TO WAVE PROPAGATION IN RANDOM MEDIA
}

\author{
JOSSELIN GARNIER
}

\begin{abstract}
In this paper a multi-scaled diffusion-approximation theorem is proved so as to unify various applications in wave propagation in random media: transmission of optical modes through random planar waveguides; time delay in scattering for the linear wave equation; decay of the transmission coefficient for large lengths with fixed output and phase difference in weakly nonlinear random media.
\end{abstract}

\section{INTRODUCTION}

Wave propagation in random media has become an extensively studied subject. In one-dimensional linear media with random inhomogeneities, localization occurs, which means in particular that the transmitted intensity decays exponentially as a function of the size of the medium. This problem has been analyzed in detail by Carmona et al. (1990).

In our paper, we consider wave reflection and transmission from a onedimensional random slab. Several quantities characterize the reflected wave; here we focus on the reflection coefficient, the phase difference and the time delay. The analysis puts into evidence the usual scales (see Knapp et al. (1989) and Papanicolaou (1988)): length of the slab, wavelength, amplitude and correlation radius of the random perturbations. We study the asymptotic behavior of the scattered wave in the framework introduced by Papanicolaou based on the separation of these scales. The fluctuations of the random coefficients are on a small scale so that we actually deal with diffusion-approximation problems. However we consider here situations where many scaled quantities play a role, so that we need to prove, then to use general multi-scaled diffusion-approximation theorems. Indeed, the study of planar waveguides depends on the above quantities, but also on the thickness of the core. The time delay depends not only on the high carrier frequency of the wave packet, but also on its bandwidth. Finally the amplitude of the nonlinear term is an essential requirement for the study of the behavior of the transmittivity for the nonlinear wave equation. The action of nonlinearity seems to be opposite to that of disorder. Nonlinearity may change the dependence of the transmission coefficient on the length, that still tends to zero as the size of the medium increases, but following a

URL address of the journal: http://www.emath.fr/ps/

Received by the journal March 18, 1996. Revised January 23, 1997. Accepted for publication February 14, 1997.

(C) Société de Mathématiques Appliquées et Industrielles. Typeset by IATEX. 
power law (see Desvillard et al. (1986) and Knapp et al. (1989)) instead of the exponential behavior observed in the linear case.

The organization of the paper is as follows: The first section is devoted to the transmission of optical modes through a random metallic planar waveguide. In Section 3 we refine some results of Faris et al. (1994) concerning the time delay in scattering for the linear wave equation with a random index of refraction. The last sections deal with the random nonlinear wave equation. In Section 4 we study the nonlinear fixed output problem, i.e. the transmission problem with a fixed outgoing intensity, which is simpler than the fixed input problem, since there are unique values of input intensity and transmittivity for a given output intensity. In Section 5 we deal with the phase difference of the reflected wave. Finally the appendix is devoted to the statements and the proofs of multi-scaled diffusion-approximation theorems.

\section{Propagation in MEtallic Planar WAVEguides}

In this section we study propagation of optical modes in dielectric films with thicknesses comparable to the wavelength. The main idea of a waveguide is to guide a beam of light by employing a variation of the index of refraction in the transverse direction so as to cause the light to travel along a well-defined channel. The dependence of the index of refraction on the transverse direction may be continuous or discontinuous (we shall consider a particular case of the discontinuous situation), but the essential element is that the index of refraction is maximal in the channel along which one whishes to guide the light.

We shall consider the basic problem of TE (transversal electric) mode propagation in slab dielectric waveguides (see Collins (1960)). Indeed the basic features of the behavior of dielectric waveguide can be extracted from a planar model in which no variation exists in one direction (say $y$ ). Channel waveguides with axis $z$, in which the waveguide dimensions are finite in both the $x$ and $y$ directions, approach the behavior of a planar waveguide when one dimension is much larger than the other (see Groell (1968)). Even when this is not the case, most of the phenomena of interest are only modified in a simply quantitative way when going from a planar waveguide to a channel waveguide. This fact combined with the great mathematical simplification of the one-dimensional case has led us to consider here planar waveguides.

We assume that the slab waveguide has thickness $2 a$ and is located in the region $x \in[-a, a]$. Its axis is $z$ and the slab is infinite in the $y$-direction. The slab is switched between two metallic slabs for $x>a$ and for $x<-a$. We shall restrict ourselves to the $y$-independent case and consider waves which depend only on $x$ and $z$.

We begin by studying the properties of guided modes in a perfect waveguide, whose core has a homogeneous index of refraction equal to $n_{0}$. A mode of a dielectric waveguide is a monochromatic wave $\mathbf{E}(t, \mathbf{r})=\mathbf{E}(\mathbf{r}) e^{-i \omega t}$ solution of the wave equation

$$
\Delta \mathbf{E}(\mathbf{r})+k_{0}^{2} n^{2}(\mathbf{r}) \mathbf{E}(\mathbf{r})=0
$$


where $k_{0}=\omega / c$ is the vacuum wavenumber. The solution satisfies continuity conditions of the tangential components of the field at the dielectric interfaces. Limiting ourselves to waves with phase front normal to the waveguide axis $z$, we have $\mathbf{E}(\mathbf{r})=\mathbf{E}(x) e^{i \beta z}$.

We are looking for $\mathrm{TE}$ modes $\mathbf{E}=\left(0, E_{y}, 0\right)$ with field component $E_{y}=$ $E(x) e^{i \beta z}$. The scalar field $E$ satisfies

$$
\frac{\partial^{2} E}{\partial x^{2}}+\left(k^{2}-\beta^{2}\right) E=0,
$$

where $k$ is the homogeneous wavenumber $k=n_{0} k_{0}$. In the metallic slabs $x>a$ and $x<-a$ the electric field is zero. Because of the need to match $E_{y}$ at $x=-a$ and $x=a$ the field $E$ solution of (2.2) satisfies the boundary conditions $E(-a, z)=E(a, z)=0$. There exists solutions only for some values of $\beta$. Thus the metallic planar waveguide can only support a finite number of confined TE modes, $E_{j}(x, z)=R_{j}(x) e^{i \beta_{j} z}$, where

$$
R_{j}(x)= \begin{cases}\cos \left(\frac{j \pi x}{2 a}\right), & \text { if } j \text { is odd } \\ \sin \left(\frac{j \pi x}{2 a}\right), & \text { if } j \text { is even, }\end{cases}
$$

and $\beta_{j}$ satisfies the dispersion relation

$$
\beta_{j}^{2}+\frac{\pi^{2} j^{2}}{4 a^{2}}=k^{2}
$$

There exists $N$ guided modes, where $N$ is the integer that satisfies

$$
\frac{2 a k}{\pi}-1 \leq N<\frac{2 a k}{\pi}
$$

We shall assume that a monochromatic guided wave is incoming from the left through a perfect waveguide and has the form $(x<0)$ :

$$
E_{i n}(x, z)=\sum_{j=1}^{N} R_{j}(x) E_{j}(z), \quad E_{j}(z)=B_{j 0} e^{i \beta_{j} z},
$$

where $\beta_{j}$ is the positive solution of (2.4) and $B_{j_{0}}$ is the decomposition of the incident wave $E_{i n}$ on the $j$-th mode. This wave is scattered by a perturbed waveguide occupying the interval $\left[0, L^{\varepsilon}\right]$, so that the total field is constituted of the sum of the incident wave (2.6) and the reflected wave in the region $x<0$, and of the transmitted wave in the region $x>L^{\varepsilon}$, where the waveguide is unperturbed. We shall consider here that the wavelength of the incident wave and the thickness of the waveguide $2 a$ are of order 1 . We assume that the medium inside the waveguide is affected by small random inhomogeneities for $z \in\left[0, L^{\varepsilon}\right]$, so that its index of refraction admits the representation:

$$
n^{\varepsilon 2}(z)=n_{0}^{2}\left(1+\varepsilon m\left(\frac{z}{\varepsilon^{r}}\right)\right),
$$

where $\varepsilon$ is a small parameter which characterizes the amplitude of the random inhomogeneities. The random coefficient $m$ which describes the inhomogeneities is assumed to be an ergodic Markov process. More exactly, we consider that the process $m$ has a unique invariant probability, under 
which it is ergodic and that it satisfies the Fredholm alternative. As a consequence, its infinitesimal generator admits an inverse on the subspace of functions centered under the invariant probability of $m$. In our paper we think at Markov processes on a compact space satisfying the Doeblin condition, however some Markov diffusion processes studied by Bouc et al. (1984) are suitable. We refer in particular to Kesten et al. (1979), Kushner (1984) and Papanicolaou et al. (1976) for sharp conditions. Throughout the paper expectations will be taken under the invariant probability of $m$ and we denote:

$$
\alpha_{c}(k)=\int_{0}^{\infty} \cos (2 k s) \mathbb{E}[m(0) m(s)] d s .
$$

Three cases appear. If $-1 \leq r<0$ (resp. $r=0, r>0$ ), the correlation radius of the inhomogeneities is much larger (resp. of the same order, much shorter) than the wavelength. The first case corresponds to a very high frequency regime, and the third one to a low frequency regime. The case $r<-1$ does not provide us with any asymptotic regime.

We shall consider a perturbed slab waveguide of length $L^{\varepsilon}=L / \varepsilon^{2+r}$ located in the region $z \in\left[0, L^{\varepsilon}\right]$. It will appear in the following that this is the judicious scale to put into evidence a macroscopic effect of the perturbations. We shall see that the fluctuations of the index of refraction induce a coupling between forward and backward modes when $r \geq 0$ and only affect the phases of the modes when $r<0$. We can now state our main result:

Proposition 2.1. The transmitted wave has the following form, for $z \geq$ $L / \varepsilon^{2+r}$ :

$$
E_{t r}(x, z)=\sum_{j=1}^{N} R_{j}(x) E_{j}\left(z, \frac{L}{\varepsilon^{2+r}}\right), \quad E_{j}\left(z, \frac{L}{\varepsilon^{2+r}}\right)=B_{j}\left(\frac{L}{\varepsilon^{2+r}}\right) e^{i \beta_{j} z},
$$

1. If $-1 \leq r<0$, then the processes $\left(B_{j}\left(L / \varepsilon^{2+r}\right)\right)_{L \geq 0}, j=1, \ldots, N$ converge weakly to $B_{j_{0}} e^{i \phi_{j}(L)}$ as $\varepsilon \rightarrow 0$,

$$
\phi_{j}(L)=\left\{\begin{array}{l}
\frac{k^{2} \sigma}{2 \beta_{j}} w_{L}, \text { if }-1<r<0, \\
\frac{k^{2}}{2 \beta_{j}} \int_{0}^{L} m(z) d z, \text { if } r=-1,
\end{array}\right.
$$

where $w$ is a standard Brownian motion independent of $j$ and $\sigma^{2}=$ $\alpha_{c}(0)$.

2. If $r \geq 0$, then the processes $\left(\left|B_{j}\left(L / \varepsilon^{2+r}\right)\right|^{2}\right)_{L \geq 0}, j=1, \ldots, N$ converge weakly to independent Markov processes $\mathcal{I}_{j}$ whose generators are

$$
\begin{gathered}
\mathcal{L}_{j}=\frac{1}{\lambda_{j}} \mathcal{I}_{j}^{2}\left(-\frac{\partial}{\partial \mathcal{I}_{j}}+\left(1-\mathcal{I}_{j}\right) \frac{\partial^{2}}{\partial \mathcal{I}_{j}^{2}}\right), \\
\text { where } \frac{1}{\lambda_{j}}=\left\{\begin{array}{l}
\frac{k^{4} \alpha_{c}\left(\beta_{j}\right)}{2 \beta_{j}^{2}}, \text { if } r=0, \\
\frac{k^{4} \alpha_{c}(0)}{2 \beta_{j}^{2}}, \text { if } r>0 .
\end{array}\right.
\end{gathered}
$$


In particular, the transmitted intensities decay exponentially with the length of the random waveguide:

$$
\lim _{L \rightarrow \infty} \frac{1}{L} \ln \mathbb{E}\left[\mathcal{I}_{j}(L)\right]=-\frac{1}{4 \lambda_{j}} .
$$

In the very high frequency regime, the perturbations only affect the phases of the optical modes by adding random phases on every mode. In the other regimes, we can observe an exponential localization of the modes, but the striking point is that each mode has its own localization length. The smaller the effective wavenumber $\beta$ is, the less the corresponding mode can penetrate in the random waveguide.

Proof. Inside the perturbed slab we expand the total field $E$ in the form

$$
E(x, z)=\sum_{j=1}^{N} R_{j}(x) E_{j}(z), \quad E_{j}(z)=A_{j}(z) e^{-i \beta_{j} z}+B_{j}(z) e^{i \beta_{j} z},
$$

where $A_{j}$ and $B_{j}$ are respectively backward (going to the right) and forward (going tot the left) optical modes. $E$ satisfies the boundary conditions at the dielectric interfaces $x=-a$ and $x=a$ and the evolution equation (2.1) which writes:

$$
\begin{gathered}
\sum_{j=1}^{N} R_{j}(x) \Psi_{j}^{\varepsilon}\left(z, A_{j}, B_{j}\right)=0, \\
\Psi_{j}^{\varepsilon}\left(z, A_{j}, B_{j}\right)=\frac{d^{2} A_{j}}{d z^{2}} e^{-i \beta_{j} z}+\frac{d^{2} B_{j}}{d z^{2}} e^{i \beta_{j} z}-2 i \beta_{j}\left(\frac{d A_{j}}{d z} e^{-i \beta_{j} z}-\frac{d B_{j}}{d z} e^{i \beta_{j} z}\right) \\
+k^{2} \varepsilon m\left(\frac{z}{\varepsilon^{r}}\right)\left(A_{j} e^{-i \beta_{j} z}+B_{j} e^{i \beta_{j} z}\right) .
\end{gathered}
$$

Integrating (2.15) with respect to $R_{l}(x) d x$, we can deduce from the orthogonality of the family $\left(R_{j}\right)_{j=1, \ldots, N}$ that

$$
\Psi_{j}^{\varepsilon}\left(z, A_{j}, B_{j}\right)=0, \text { for every } j=1, \ldots, N .
$$

The orthogonality of the modes is of great importance. It will insure that there exists only coupling between forward and backward modes, and not between $j$ and $j^{\prime}$ modes.

Let us study the equation (2.16). If the couple $\left(\tilde{A}_{j}, \tilde{B}_{j}\right)$ is a solution, then the couple $\left(A_{j}, B_{j}\right)$ defined by $A_{j}(z)=\tilde{A}_{j}(z)+f(z) e^{i \beta_{j} z}, B_{j}(z)=\tilde{B}_{j}(z)-$ $f(z) e^{-i \beta_{j} z}$, is another solution of (2.16), whatever $f$ is. Choosing $f(z)=$ $\frac{i}{2 \beta_{j}}\left(\frac{d \tilde{A}_{j}}{d z} e^{-i \beta_{j} z}+\frac{d \tilde{B}_{j}}{d z} e^{i \beta_{j} z}\right)$, we come to the conclusion that $\left(A_{j}, B_{j}\right)$ satisfies both (2.16) and the relation

$$
\frac{d A_{j}}{d z} e^{-i \beta_{j} z}+\frac{d B_{j}}{d z} e^{i \beta_{j} z}=0 .
$$

We actually do not look for every solution of (2.16) since we aim at studying $E_{j}$ defined in (2.14). So we can restrict ourselves to $\left(A_{j}, B_{j}\right)$ which satisfies both (2.16) and (2.17). Finally, injecting (2.17) in (2.16), the normalized processes $A_{j}^{\varepsilon}, B_{j}^{\varepsilon}, j=1, \ldots, N$ given by

$$
A_{j}^{\varepsilon}(z)=A_{j}\left(\frac{z}{\varepsilon^{2+r}}\right), \quad B_{j}^{\varepsilon}(z)=B_{j}\left(\frac{z}{\varepsilon^{2+r}}\right),
$$


are solutions of

$$
\begin{gathered}
\frac{d}{d z}\left(\begin{array}{c}
A_{j}^{\varepsilon}(z) \\
B_{j}^{\varepsilon}(z)
\end{array}\right)=P_{j}^{\varepsilon}(z)\left(\begin{array}{c}
A_{j}^{\varepsilon}(z) \\
B_{j}^{\varepsilon}(z)
\end{array}\right), \\
P_{j}^{\varepsilon}(z)=\frac{i k^{2}}{2 \beta_{j} \varepsilon^{1+r}} m\left(\frac{z}{\varepsilon^{2+2 r}}\right)\left(\begin{array}{cc}
-1 & -e^{i \frac{2 \beta_{j} z}{\varepsilon^{2+r}}} \\
e^{-i \frac{2 \beta_{j} z}{\varepsilon^{2+r}}} & 1
\end{array}\right) .
\end{gathered}
$$

There is no wave entering the perturbed slab at $z=L$ (in the normalized scale) and the incident wave at $z=0$ has the form (2.6), so $A_{j}^{\varepsilon}$ and $B_{j}^{\varepsilon}$ satisfy the boundary conditions

$$
A_{j}^{\varepsilon}(L)=0, \quad B_{j}^{\varepsilon}(0)=B_{j_{0}} .
$$

We aim at proving an asymptotic theorem for $B_{j}^{\varepsilon}(L)$ as $\varepsilon$ goes to 0 . Instead of working with $A_{j}^{\varepsilon}$ and $B_{j}^{\varepsilon}$, we shall use the propagator $Y_{j}^{\varepsilon}$, i.e. the matrix which satisfies:

$$
\left(\begin{array}{c}
A_{j}^{\varepsilon}(L) \\
B_{j}^{\varepsilon}(L)
\end{array}\right)=Y_{j}^{\varepsilon}(L)\left(\begin{array}{c}
A_{j}^{\varepsilon}(0) \\
B_{j}^{\varepsilon}(0)
\end{array}\right)
$$

The matrix $Y_{j}^{\varepsilon}$ is solution of the linear differential equation:

$$
\frac{d Y_{j}^{\varepsilon}}{d z}(z)=P_{j}^{\varepsilon}(z) Y_{j}^{\varepsilon}(z), \quad Y_{j}^{\varepsilon}(0)=I_{d}
$$

If $\left(a_{j}^{\varepsilon}, b_{j}^{\varepsilon}\right)$ is a solution of $(2.19)$ with the initial conditions

$$
a_{j}^{\varepsilon}(0)=1, \quad b_{j}^{\varepsilon}(0)=0
$$

then it can be readily checked that $\left(b_{j}^{\varepsilon *}, a_{j}^{\varepsilon *}\right)$ is another solution of (2.19) linearly independent of $\left(a_{j}^{\varepsilon}, b_{j}^{\varepsilon}\right)$, so we can write

$$
Y_{j}^{\varepsilon}(z)=\left(\begin{array}{cc}
a_{j}^{\varepsilon}(z) & b_{j}^{\varepsilon *}(z) \\
b_{j}^{\varepsilon}(z) & a_{j}^{\varepsilon *}(z)
\end{array}\right) .
$$

From

$$
\left(\begin{array}{c}
0 \\
B_{j}^{\varepsilon}(L)
\end{array}\right)=Y_{j}^{\varepsilon}(L)\left(\begin{array}{c}
A_{j}^{\varepsilon}(0) \\
B_{j_{0}}
\end{array}\right)
$$

we can deduce that

$$
A_{j}^{\varepsilon}(0)=-\frac{b_{j}^{\varepsilon}(L)^{*} B_{j_{0}}}{a_{j}^{\varepsilon}(L)}, \quad B_{j}^{\varepsilon}(L)=\frac{B_{j_{0}}}{a_{j}^{\varepsilon}(L)} .
$$

Since the matrix $P_{j}^{\varepsilon}$ has trace zero, the determinant of the matrix $Y_{j}^{\varepsilon}$ is constant, i.e. $\left|a_{j}^{\varepsilon}(z)\right|^{2}-\left|b_{j}^{\varepsilon}(z)\right|^{2}=1$, so that we get the energy conservation relation:

$$
\left|A_{j}^{\varepsilon}(0)\right|^{2}+\left|B_{j}^{\varepsilon}(L)\right|^{2}=\left|B_{j_{0}}\right|^{2}
$$

which means that the intensity of each incident mode has split into a transmitted intensity $\left|B_{j}^{\varepsilon}(L)\right|^{2}$ and a reflected intensity $\left|A_{j}^{\varepsilon}(0)\right|^{2}$.

So we have transformed the boundary value problem $(2.19),(2.21)$ into an initial value problem $(2.19),(2.24)$. In order to be allowed to apply the diffusion-approximation theorems, we have to take care to consider separately the real and imaginary parts of each coefficient $a_{j}^{\varepsilon}$ and $b_{j}^{\varepsilon}$, so that we actually deal with a system with $4 N$ linear differential equations. Denoting 
$X_{4 j+1}^{\varepsilon}=\operatorname{Re}\left(a_{j}^{\varepsilon}\right), X_{4 j+2}^{\varepsilon}=\operatorname{Im}\left(a_{j}^{\varepsilon}\right), X_{4 j+3}^{\varepsilon}=\operatorname{Re}\left(b_{j}^{\varepsilon}\right)$ and $X_{4 j+4}^{\varepsilon}=\operatorname{Im}\left(b_{j}^{\varepsilon}\right)$,

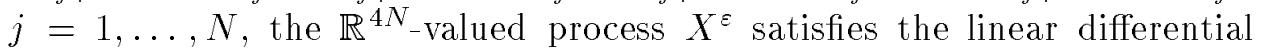
equation

$$
\frac{d X^{\varepsilon}(z)}{d z}=\frac{1}{\varepsilon^{1+r}} F\left(m\left(\frac{z}{\varepsilon^{2+2 r}}\right), \frac{z}{\varepsilon^{2+r}}\right) X^{\varepsilon}(z),
$$

with the initial conditions $X_{4 j+j^{\prime}}^{\varepsilon}(0)=1$ if $j^{\prime}=1, X_{4 j+j^{\prime}}^{\varepsilon}(0)=0$ if $j^{\prime}=$ $2,3,4$, where

$$
F(m, h)=\oplus_{j=1}^{N} \frac{k^{2} m}{2 \beta_{j}}\left(\begin{array}{cccc}
0 & 1 & \sin \left(2 \beta_{j} h\right) & \cos \left(2 \beta_{j} h\right) \\
-1 & 0 & -\cos \left(2 \beta_{j} h\right) & \sin \left(2 \beta_{j} h\right) \\
\sin \left(2 \beta_{j} h\right) & -\cos \left(2 \beta_{j} h\right) & 0 & -1 \\
\cos \left(2 \beta_{j} h\right) & \sin \left(2 \beta_{j} h\right) & 1 & 0
\end{array}\right)
$$

Then we transform $\varepsilon^{1+r} \mapsto \varepsilon$, so that the system writes now as an usual diffusion-approximation problem. The application of Theorem 2-7 Papanicolaou et al. (1976) (resp. Theorem 2-8 Papanicolaou et al. (1976)) provides us with the desired result for the case $r=0$ (resp. $r>0$ ). The case $r<0$ can be deduced from Theorem 6.2 in the appendix.

\section{TIME DELAY}

We aim at studying the time delay of a wave packet scattered by a onedimensional random medium. The time delay is the difference between times spent by the wave packet in the perturbed region and in the homogeneous space. For instance, a positive time delay means that the wave packet has spent more time in the scattering region than the one it would have spent in homogeneous space.

We consider a wave packet reflected by a one-dimensional random medium located in the region $[0, L]$. The wave packet is assumed to satisfy the linear wave equation:

$$
\frac{n^{\varepsilon 2}(x)}{c^{2}} \frac{\partial^{2} E}{\partial t^{2}}=\frac{\partial^{2} E}{\partial x^{2}}
$$

where the index of refraction $n^{\varepsilon}$ admits the representation

$$
n^{\varepsilon 2}(x)=n_{0}^{2}\left(1+m^{\varepsilon}(x)\right), \quad m^{\varepsilon}(x)=\varepsilon m\left(\frac{x}{\varepsilon^{2}}\right) .
$$

$m$ is an ergodic Markov process, which describes the perturbations of the index of refraction. Their amplitude is of order $\varepsilon$ and their correlation radius is of order $\varepsilon^{2}$, which is small compared to the length $L$ of the slab.

We consider the matched medium boundary condition. It is assumed that a wave packet is incident on the random slab $[0, L]$ from a homogeneous medium with index $n_{0}$ occupying $x<0$ :

$$
u_{i}(t, x)=\int_{0}^{\infty} e^{i\left(k x-c k t / n_{0}\right)} \hat{u}_{i}(k) d k,
$$

where $\hat{u}_{i}$ is the spectrum of the incident wave packet. The boundary condition at $x=L$ is for termination of the random slab by a uniform medium. We will have total reflection for large length $L$ because the wave cannot penetrate to infinite depth. Indeed the reflection coefficient converges exponentially fast to 1 which follows from Furstenberg's theorem (see Carmona (1985)). So it is convenient to analyze the problem with a totally reflecting 
termination at $x=L$, so that the number of degrees of freedom is reduced by one. Thus the wave packet is scattered and the reflected wave has the following form for large $t$ :

$$
u_{r}^{\varepsilon}(t, x)=\int_{0}^{\infty} R^{\varepsilon}(k) e^{i\left(-k x-c k t / n_{0}\right)} \hat{u}_{i}(k) d k,
$$

where $R^{\varepsilon}(k)$ is the reflection coefficient associated to the homogeneous wavenumber $k$. Because of the reflecting boundary condition, the modulus of $R^{\varepsilon}(k)$ is equal to 1 so that $R^{\varepsilon}(k)=e^{i \theta^{\varepsilon}(k)+i \theta_{0}(k)}$. The phase $\theta_{0}(k)=2 k L+\pi$ is related to the homogeneous part of the index of refraction. If the slab $[0, L]$ were occupied by a homogeneous medium, the reflected wave would be exactly:

$$
u_{r 0}(t, x)=\int_{0}^{\infty} e^{i\left(-k x-c k t / n_{0}\right)} e^{i \theta_{0}(k)} \hat{u}_{i}(k) d k .
$$

The phase $\theta^{\varepsilon}(k)$ characterizes the influence of the fluctuations of the index of refraction onto the reflected wave. In particular the time delay is given by

$$
T^{\varepsilon}\left(u_{i}\right)=\frac{n_{0}}{c} \int_{0}^{\infty} \tau^{\varepsilon}(k)\left|\hat{u}_{i}\right|^{2}(k) d k,
$$

where $\tau^{\varepsilon}(k):=\frac{d \theta^{\varepsilon}}{d k}(k)$. A rigorous proof may be derived from Jauch et al. (1972), but we can heuristically put into evidence (3.6) (cf Faris et al. (1994)). Assume that $\hat{u}_{i}$ is concentrated near some wavenumber $k_{c}$. By expanding $R^{\varepsilon}(k)$ about $k_{c}$ in (3.4), we find that the reflected wave is, up to a multiplicative constant:

$$
u_{r}^{\varepsilon}(t, x) \simeq \int_{0}^{\infty} e^{i\left(-k x-c k\left(t-n_{0} \tau^{\varepsilon}\left(k_{c}\right) / c\right) / n_{0}\right)} e^{i \theta_{0}(k)} \hat{u}_{i}(k) d k
$$

By such a way we can understand why $n_{0} \tau^{\varepsilon}\left(k_{c}\right) / c$ represents the time delay at frequency $k_{c}$.

Faris et al. (1994) studied the one-dimensional distribution of $\tau^{\varepsilon}(k)$ for some $k$ and considered the limit case $\varepsilon \rightarrow 0$, and then $k \rightarrow \infty$. We first prove that we can deal with simultaneous limits when $k$ is of order $\varepsilon^{-2}$, i.e. when the wavelength and the correlation radius of the perturbations are of the same order.

Proposition 3.1. The process $\left(\tau^{\varepsilon}\left(k / \varepsilon^{2}, L\right)\right)_{L>0}$ converges in distribution in $\mathbf{C}^{0}([0, \infty), \mathbb{R})$ to the inhomogeneous Markov process $(\tau(L))_{L>0}$ whose infinitesimal generator is:

$$
\mathcal{L}_{k}=\frac{1}{2} \alpha_{c}(k) k^{2}(\tau+2 L)^{2} \frac{\partial^{2}}{\partial \tau^{2}}
$$

where $\alpha_{c}(k)$ has been defined by (2.8). In particular $\tau(L)$ is a centered process, whose variance is:

$$
\mathbb{E}\left[\tau(L)^{2}\right]=\frac{8}{\alpha_{c}(k)^{2} k^{4}}\left(e^{\alpha_{c}(k) k^{2} L}-1-\alpha_{c}(k) k^{2} L-\frac{1}{2}\left(\alpha_{c}(k) k^{2} L\right)^{2}\right) .
$$

Its distribution can be represented as follows:

$$
\tau(L)=X(L)-2 L, X(L)=\frac{2}{\alpha_{c}(k) k^{2}} \int_{0}^{\alpha_{c}(k) k^{2} L} e^{w_{s}-\frac{1}{2} s} d s,
$$


where $w$ is a standard Brownian motion.

Proof. Following Papanicolaou (1988), we find that the phase $\theta^{\varepsilon}$ satisfies:

$$
\frac{d \theta^{\varepsilon}}{d L}=k^{\varepsilon}\left(1-\cos \left(\theta^{\varepsilon}+2 k^{\varepsilon} L\right)\right) m^{\varepsilon}(L), \quad \theta^{\varepsilon}(0)=0 .
$$

Differentiating with respect to the wavenumber yields the equation which governs the evolution of $\tau^{\varepsilon}$ :

$$
\frac{d \tau^{\varepsilon}}{d L}=\left(1-\cos \left(\theta^{\varepsilon}+2 k^{\varepsilon} L\right)\right) m^{\varepsilon}(L)+k^{\varepsilon} \sin \left(\theta^{\varepsilon}+2 k^{\varepsilon} L\right)\left(\tau^{\varepsilon}+2 L\right) m^{\varepsilon}(L) .
$$

Introducing the different scales in the evolution equation, the process $\left(\theta^{\varepsilon}, \tau^{\varepsilon}\right)$ satisfies, up to negligible terms of order $\varepsilon$ :

$$
\begin{cases}\frac{d \theta^{\varepsilon}}{d L}=\frac{k}{\varepsilon}\left(1-\cos \left(\theta^{\varepsilon}+\frac{2 k L}{\varepsilon^{2}}\right)\right) m\left(\frac{L}{\varepsilon^{2}}\right), & \theta^{\varepsilon}(0)=\pi, \\ \frac{d \tau^{\varepsilon}}{d L}=\frac{k}{\varepsilon} \sin \left(\theta^{\varepsilon}+\frac{2 k L}{\varepsilon^{2}}\right)\left(\tau^{\varepsilon}+2 L\right) m\left(\frac{L}{\varepsilon^{2}}\right), & \tau^{\varepsilon}(0)=0 .\end{cases}
$$

The application of Theorem 2-7 Papanicolaou et al. (1976) completes the proof.

The above analysis leads to the asymptotic distribution of $\tau^{\varepsilon}(k)$ for a fixed wavenumber. However it is necessary to find the joint distribution of $\tau^{\varepsilon}(k)$ for several wavenumbers $k$ in order to study the behavior of the time delay $T^{\varepsilon}\left(u_{i}\right)$ for an incident wave packet $u_{i}$. For instance the estimation of the $p$-th moment of $T^{\varepsilon}\left(u_{i}\right)$ requires the computation of the joint distribution of the $p$-uplet $\left(\tau^{\varepsilon}\left(k_{1}\right), \ldots, \tau^{\varepsilon}\left(k_{p}\right)\right)$, since

$$
\mathbb{E}\left[T^{\varepsilon}\left(u_{i}\right)^{p}\right]=\frac{n_{0}^{p}}{c^{p}} \int \mathbb{E}\left[\tau^{\varepsilon}\left(k_{1}\right) \ldots \tau^{\varepsilon}\left(k_{p}\right)\right]\left|\hat{u}_{i}\right|^{2}\left(k_{1}\right) \ldots\left|\hat{u}_{i}\right|^{2}\left(k_{p}\right) d k_{1} \ldots d k_{p} .
$$

We now state the main result of this section about the convergence of the time delay. We assume that an incident wave (3.3) with a high carrier frequency $k_{c} / \varepsilon^{2}$ and a bandwidth of order $\varepsilon^{a-2}$ is incoming from the right.

Proposition 3.2. Let us assume that the Fourier transform of the incident wave $\hat{u}_{i}^{\varepsilon}$ has the form

$$
\hat{u}_{i}^{\varepsilon}(k)=\hat{\psi}\left(\frac{\varepsilon^{a} k-k_{c}}{\varepsilon^{2}}\right),
$$

where $\hat{\psi}$ is a continuous function with compact support.

- If $0 \leq a<2$, then the time delay $\left(T^{\varepsilon}\left(u_{i}^{\varepsilon}\right)(L)\right)_{L \geq 0}$ converges weakly to the process 0 .

- If $a=2$, then $\left(T^{\varepsilon}\left(u_{i}^{\varepsilon}\right)(L)\right)_{L \geq 0}$ converges weakly to a centered and continuous process with variance:

$$
\mathbb{E}\left[T^{2}(L)\right]=\frac{n_{0}^{2}}{c^{2}} \frac{8 e^{\alpha_{c}\left(k_{c}\right) k_{c}^{2} L}}{\pi \alpha_{c}\left(k_{c}\right)^{2} k_{c}^{4} L}\left(\int|\hat{\psi}(k)|^{4} d k\right)+\underset{L \rightarrow \infty}{o}\left(\frac{e^{\alpha_{c}\left(k_{c}\right) k_{c}^{2} L}}{\alpha_{c}\left(k_{c}\right) k_{c}^{2} L}\right) .
$$


- If $a>2$, then $\left(\varepsilon^{a-2} T^{\varepsilon}\left(u_{i}^{\varepsilon}\right)(L)\right)_{L \geq 0}$ converges weakly to a centered and continuous process with variance:

$$
\mathbb{E}\left[T^{2}(L)\right]=\frac{n_{0}^{2}}{c^{2}} \frac{8 e^{\alpha_{c}\left(k_{c}\right) k_{c}^{2} L}}{\alpha_{c}\left(k_{c}\right)^{2} k_{c}^{4}}\left(\int|\hat{\psi}(k)|^{2} d k\right)^{2}+\underset{L \rightarrow \infty}{o}\left(e^{\alpha_{c}\left(k_{c}\right) k_{c}^{2} L}\right) .
$$

Proof. We assume that the support of $\hat{\psi}$ is contained in $(-N, N)$. We denote by $\tau^{\varepsilon}(L, h)$ the process $\left.\tau^{\varepsilon}\left(L, k^{\varepsilon}\right)\right|_{k^{\varepsilon}=\left(k_{c}+\varepsilon^{a} h\right) / \varepsilon^{2}}$. From the expression (3.6) of the time delay, if we prove that $\tau^{\varepsilon}(L, h)$ converges weakly as a process in $\mathrm{C}^{0}\left([0, \infty), L^{2}(-N, N)\right)$, then it will follow that $T^{\varepsilon}\left(u_{i}^{\varepsilon}\right)$ converges weakly in $\mathbf{C}^{0}([0, \infty), \mathbb{R})$.

Step 1. Convergence of the finite-dimensional Distributions of $\tau^{\varepsilon}$. First we deal with the two-dimensional distributions of the process $\tau^{\varepsilon}$. We aim at finding the asymptotic joint distribution of the couple $\left(\tau_{1}^{\varepsilon}(L), \tau_{2}^{\varepsilon}(L)\right)_{L \geq 0}$, where $\tau_{i}^{\varepsilon}=\tau^{\varepsilon}\left(L, k_{i}^{\varepsilon}\right)$ and $k_{i}^{\varepsilon}$ are wavenumbers given by $k_{i}^{\varepsilon}=\left(k_{c}+\varepsilon^{a} h_{i}\right) / \varepsilon^{2}$. If we denote $\left.\theta(k, L)\right|_{k=k_{i}^{\varepsilon}}$ by $\theta_{i}^{\varepsilon}$ then we find that $\left(\theta_{1}^{\varepsilon}(L), \theta_{2}^{\varepsilon}(L), \tau_{1}^{\varepsilon}(L), \tau_{2}^{\varepsilon}(L)\right)_{L \geq 0}$ satisfies:

$$
\begin{cases}\frac{d \theta_{1}^{\varepsilon}}{d L}=\frac{k_{c}+\varepsilon^{a} h_{1}}{\varepsilon}\left(1-\cos \left(\theta_{1}^{\varepsilon}+\frac{2 k_{c} L}{\varepsilon^{2}}+\frac{2 h_{1} L}{\varepsilon^{2-a}}\right)\right) m\left(\frac{L}{\varepsilon^{2}}\right), & \theta_{1}^{\varepsilon}(0)=0, \\ \frac{d \theta_{2}^{\varepsilon}}{d L}=\frac{k_{c}+\varepsilon^{a} h_{2}}{\varepsilon}\left(1-\cos \left(\theta_{2}^{\varepsilon}+\frac{2 k_{c} L}{\varepsilon^{2}}+\frac{2 h_{2} L}{\varepsilon^{2-a}}\right)\right) m\left(\frac{L}{\varepsilon^{2}}\right), & \theta_{2}^{\varepsilon}(0)=0, \\ \frac{d \tau_{1}^{\varepsilon}}{d L}=\frac{k_{c}+\varepsilon^{a} h_{1}}{\varepsilon} \sin \left(\theta_{1}^{\varepsilon}+\frac{2 k_{c} L}{\varepsilon^{2}}+\frac{2 h_{1} L}{\varepsilon^{2-a}}\right)\left(\tau_{1}^{\varepsilon}+2 L\right) m\left(\frac{L}{\varepsilon^{2}}\right), & \tau_{1}^{\varepsilon}(0)=0, \\ \frac{d \tau_{2}^{\varepsilon}}{d L}=\frac{k_{c}+\varepsilon^{a} h_{2}}{\varepsilon} \sin \left(\theta_{2}^{\varepsilon}+\frac{2 k_{c} L}{\varepsilon^{2}}+\frac{2 h_{2} L}{\varepsilon^{2-a}}\right)\left(\tau_{2}^{\varepsilon}+2 L\right) m\left(\frac{L}{\varepsilon^{2}}\right), & \tau_{2}^{\varepsilon}(0)=0 .\end{cases}
$$

- If $0<a<2$, then we get by applying Theorem 6.1 in the appendix in the case $A=2 k_{c}, B_{1}=1$ and $B_{j}=0, j \geq 2$ that the process $\left(\tau_{1}^{\varepsilon}, \tau_{2}^{\varepsilon}\right)$ converges to $\left(\tau_{1}, \tau_{2}\right)$, where $\tau_{1}$ and $\tau_{2}$ are independent Markov process with the same generator $\mathcal{L}_{k_{c}}$ given by (3.8).

- If $a=0$, then by the standard Theorem 2-7 Papanicolaou et al. (1976) the process $\left(\tau_{1}^{\varepsilon}, \tau_{2}^{\varepsilon}\right)$ converges to $\left(\tau_{1}, \tau_{2}\right)$, where $\tau_{1}$ and $\tau_{2}$ are independent Markov process with generators $\mathcal{L}_{k_{c}+h_{1}}$ and $\mathcal{L}_{k_{c}+h_{2}}$ respectively.

- If $a>2$, then $\left(\tau_{1}^{\varepsilon}, \tau_{2}^{\varepsilon}\right)$ converges to $(\tau, \tau)$, where $\tau$ is a Markov process with generator $\mathcal{L}_{k_{c}}$.

- If $a=2$, then $\left(\theta_{1}^{\varepsilon}(L), \theta_{2}^{\varepsilon}(L), \tau_{1}^{\varepsilon}(L), \tau_{2}^{\varepsilon}(L)\right)_{L>0}$ converges in distribution in $\mathbf{C}^{0}\left([0, \infty), \mathbb{R}^{4}\right)$ to the Markov process $\left(\theta_{1}(L), \theta_{2}(L), \tau_{1}(L), \tau_{2}(L)\right)_{L \geq 0}$ whose infinitesimal generator is $\left(h=h_{2}-h_{1}\right)$ :

$$
\begin{aligned}
\mathcal{L}=\alpha_{c}(0) k_{c}^{2}\left(\frac{\partial}{\partial \theta_{1}}\right. & \left.+\frac{\partial}{\partial \theta_{2}}\right)^{2} \\
+ & \frac{1}{2} \alpha_{c}\left(k_{c}\right) k_{c}^{2}\left(\frac{\partial^{2}}{\partial \theta_{1}^{2}}+\frac{\partial^{2}}{\partial \theta_{2}^{2}}+2 \cos \left(\theta_{2}-\theta_{1}+2 h L\right) \frac{\partial^{2}}{\partial \theta_{1} \theta_{2}}\right) \\
+ & \frac{1}{2} \alpha_{c}\left(k_{c}\right) k_{c}^{2}\left(\left(\tau_{1}+2 L\right)^{2} \frac{\partial^{2}}{\partial \tau_{1}^{2}}+\left(\tau_{2}+2 L\right)^{2} \frac{\partial^{2}}{\partial \tau_{2}^{2}}\right. \\
& \left.+2\left(\tau_{1}+2 L\right)\left(\tau_{2}+2 L\right) \cos \left(\theta_{2}-\theta_{1}+2 h L\right) \frac{\partial^{2}}{\partial \tau_{1} \tau_{2}}\right) .
\end{aligned}
$$


Papanicolaou (1988) proved the convergence of the couple $\left(\theta_{1}^{\varepsilon}(L), \theta_{2}^{\varepsilon}(L)\right)_{L \geq 0}$, and also that the process $\left(\theta_{2}(L)-\theta_{1}(L)+2 h L \bmod 2 \pi\right)_{L \geq 0}$ admits an invariant probability measure $m_{h}$ which satisfies:

$$
\langle\cos \phi\rangle_{m_{h}}=\frac{2|h|}{\alpha_{c}\left(k_{c}\right) k_{c}^{2}} \int_{0}^{\infty} \exp \left(-\frac{2|h| z}{\alpha_{c}\left(k_{c}\right) k_{c}^{2}}\right) \frac{z^{2}}{z^{2}+4} d z .
$$

By a straightforward generalization of the above results we can show that the finite-dimensional distributions $\left(\tau^{\varepsilon}\left(L, h_{1}\right), \ldots, \tau^{\varepsilon}\left(L, h_{n}\right)\right)_{L \geq 0}$ converge in $\mathbf{C}^{0}\left([0, \infty), \mathbb{R}^{n}\right)$.

It remains to prove the tightness of $\tau^{\varepsilon}(L, h)$ in $\mathbf{C}^{0}\left([0, \infty), L^{2}(-N, N)\right)$. We will only deal with the most delicate case $a=2$. In fact we shall show that $\tau^{\varepsilon}(L, h)$ is tight in $\mathbf{D}\left([0, \infty), L^{2}(-N, N)\right)$ and we shall conclude by checking that the weak limit is unique and belongs to $\mathrm{C}^{0}\left([0, \infty), L^{2}(-N, N)\right)$.

We begin by stating some standard tightness criteria (see Metivier (1984)).

LEMMA 3.3. Let $(E, d)$ be a metric space, and $X^{\varepsilon}$ a process with paths in $\mathbf{D}([0, \infty), E)$. If for every $t$ in a dense subset of $\mathbb{R}^{+}$the family $\left(X^{\varepsilon}(t)\right)_{\varepsilon \in(0,1]}$ is tight in $E$ and $X^{\varepsilon}$ satisfies the Aldous property:

For any $M>0, \eta>0, \lambda>0$, there exists $\delta>0$ such that $[A] \operatorname{limsupsup}_{\varepsilon \rightarrow 0} \sup _{0<\theta<\delta} \mathbb{P}\left(\left\|X^{\varepsilon}(T+\theta)-X^{\varepsilon}(T)\right\|>\lambda\right)<\eta$

where $T$ is a stopping time and $\sup _{T}$ is the sup over all such $T \leq M$, then the family $\left(X^{\varepsilon}\right)_{\varepsilon \in(0,1]}$ is tight in $\mathbf{D}([0, \infty), E)$.

LemMa 3.4. Let $H$ be a Hilbert space and $H_{n}$ be an increasing sequence of finite-dimensional spaces in $H$ such that, for any $h \in H, \lim _{n \rightarrow \infty} \pi_{H_{n}} h=h$. Let $Y^{\varepsilon}$ be a $H$-valued process.

$Y^{\varepsilon}$ is tight if and only if for any $\eta>0$ and $\lambda>0$, there exists $\rho_{\eta}$ and $a$ subspace $H_{\eta, \lambda}$ such that

$$
\sup _{\varepsilon \in(0,1]} \mathbb{P}\left(\left\|Y^{\varepsilon}\right\| \geq \rho_{\eta}\right) \leq \eta \text { and } \sup _{\varepsilon \in(0,1]} \mathbb{P}\left(d\left(Y^{\varepsilon}, H_{\eta, \lambda}\right)>\lambda\right) \leq \eta .
$$

Here we will take $H=L^{2}(-N, N)$ and $H_{n}$ the set of all the simple functions of the form

$$
h(t)=\sum_{k=-N n}^{N n-1} \alpha_{k} \mathbb{1}_{t \in[k / n, k+1 / n)}, \quad\left(\alpha_{k}\right)_{k=-N n, \ldots, N n-1} \in \mathbb{R}^{2 N n} .
$$

If $g \in \mathbf{C}^{0}([-N, N], \mathbb{R})$, we denote by $\pi_{n}(g)$ the simple function in $H_{n}$ defined by $g\left(\frac{[n t]}{n}\right)$.

Step 2. $\left(\tau^{\varepsilon}(L, h)\right)_{h \in(-N, N)}$ IS Tight in $L^{2}(-N, N)$ For Every $L$. By Lemma 3.4 it is sufficient to check that $\left(\tau^{\varepsilon}(L, h)\right)_{h \in(-N, N)}$ fulfills (3.15). The following criteria $(3.16,3.17)$ insures that $\left(\tau^{\varepsilon}(L, h)\right)_{h \in(-N, N)}$ fulfills $(3.15)$ :

$$
\begin{gathered}
\lim _{\delta \rightarrow 0} \limsup _{\varepsilon \rightarrow 0} \mathbb{E}\left[\sup _{\left|h-h^{\prime}\right| \leq \delta,|h| \leq N,\left|h^{\prime}\right| \leq N}\left|\tau^{\varepsilon}(L, h)-\tau^{\varepsilon}\left(L, h^{\prime}\right)\right|^{2}\right]=0, \\
\limsup _{\substack{\sup _{0 \rightarrow 0} \\
|h| \leq N}} \mathbb{E}\left[\left|\tau^{\varepsilon}(L, h)\right|^{2}\right]<\infty .
\end{gathered}
$$


If $g \in \mathbf{C}^{0}([-N, N], \mathbb{R})$ and $\sup _{\left|x-x^{\prime}\right| \leq 1 / n}\left|g(x)-g\left(x^{\prime}\right)\right| \leq \delta$, then we obviously get that $\left\|g-\pi_{n}(g)\right\|_{L^{2}(-N, N)} \leq \sqrt{2 N} \delta$, which implies $d\left(g, H_{n}\right) \leq \sqrt{2 N} \delta$.

First we check by the perturbed function method (see estimates (6.6) and (6.7) in Section 6) that the second moment of $\tau^{\varepsilon}(L, h)$ is uniformly bounded with respect to $\varepsilon \in(0,1], h \in[-N, N]$ and $L \in[0, M]$. Indeed, denoting $f(\tau)=\tau^{2}$,

$$
\begin{aligned}
\mathbb{E}\left[f\left(\tau^{\varepsilon}(L)\right)\right]= & \mathbb{E}\left[f\left(\tau^{\varepsilon}(L)\right)-f^{\varepsilon}(L)\right] \\
& +\int_{0}^{L} \mathbb{E}\left[\mathcal{L}_{s}^{\varepsilon} f^{\varepsilon}(s)-\mathcal{L}_{s} f\left(\tau^{\varepsilon}(s)\right)\right]+\int_{0}^{L} \mathbb{E}\left[\mathcal{L}_{s} f\left(\tau^{\varepsilon}(s)\right)\right],
\end{aligned}
$$

where $\mathcal{L}_{L} f(\tau)=\alpha_{c}(k) k^{2}(\tau+2 L)^{2}$ and the perturbed test functions $f^{\varepsilon}$ are built according to the procedure described in the proof of Theorem 6.1. The functions satisfy in this particular case $\left|f\left(\tau^{\varepsilon}(L)\right)-f^{\varepsilon}(L)\right| \leq$ $\varepsilon K_{L}\left(1+f\left(\tau^{\varepsilon}(L)\right)\right)$ and $\left|\mathcal{L}_{L} f\left(\tau^{\varepsilon}(L)\right)-\mathcal{L}_{L}^{\varepsilon} f^{\varepsilon}(L)\right| \leq \varepsilon K_{L}\left(1+f\left(\tau^{\varepsilon}(L)\right)\right)$. This yields (3.17) from Gronwall's inequality.

On the other hand, from the obvious estimate $\left|\tau^{\varepsilon}(L, h)-\tau^{\varepsilon}\left(L, h^{\prime}\right)\right| \leq$ $\left|h-h^{\prime}\right| \sup _{|h| \leq N}\left|\frac{\partial \tau^{\varepsilon}(L, h)}{\partial h}\right|$ and from Sobolev's imbedding $\mathbf{W}^{1,2}([-N, N]) \hookrightarrow$ $\mathrm{C}^{0}([-N, N], \mathbb{R})$, we get that there exists a constant $C_{N}$ such that

$$
\begin{aligned}
& \mathbb{E}\left[\sup _{\left|h-h^{\prime}\right| \leq \delta,|h| \leq N,\left|h^{\prime}\right| \leq N}\left|\tau^{\varepsilon}(L, h)-\tau^{\varepsilon}\left(L, h^{\prime}\right)\right|^{2}\right] \\
& \leq C_{N} \delta^{2} \sup _{|h| \leq N} \mathbb{E}\left[\left|\frac{\partial \tau^{\varepsilon}(L, h)}{\partial h}\right|^{2}+\left|\frac{\partial^{2} \tau^{\varepsilon}(L, h)}{\partial h^{2}}\right|^{2}\right],
\end{aligned}
$$

which yields (3.16) since the second moments of $\frac{\partial \tau^{\varepsilon}(L, h)}{\partial h}$ and $\frac{\partial^{2} \tau^{\varepsilon}(L, h)}{\partial h^{2}}$ are uniformly bounded with respect to $\varepsilon \in(0,1], h \in[-N, N]$ and $L \in[0, M]$ (again by the perturbed test function method). So we have stated the tightness of $\left(\tau^{\varepsilon}(L, h)\right)_{h \in(-N, N)}$ in $L^{2}(-N, N)$ for any $L$.

Step 3. $\tau^{\varepsilon}$ IS TIGHT In $\mathbf{D}\left([0, \infty), L^{2}(-N, N)\right)$. The following criteria insures that the process $\tau^{\varepsilon}$ satisfies the Aldous property [A] if we take $E=$ $L^{2}(-N, N)$ :

$$
\lim _{\delta \rightarrow 0} \limsup _{\varepsilon \rightarrow 0} \sup _{|h|<N} \sup _{T} \sup _{0<\theta<\delta} \mathbb{E}\left[1 \wedge\left|\tau^{\varepsilon}(T+\theta, h)-\tau^{\varepsilon}(T, h)\right|^{2}\right]=0,
$$

where $\sup _{T}$ is taken over all the stopping times $T$ such that $T \leq M$. Using Theorems III-3 and 4 Kushner (1984) and the estimates of the perturbed test function (6.6) and (6.7), we easily establish that $\tau^{\varepsilon}$ satisfies (3.18). Combining this result with Lemma 3.3 yields the tightness of $\tau^{\varepsilon}$ as a process in $\mathbf{D}\left([0, \infty), L^{2}(-N, N)\right)$.

Step 4. Convergence of the process $T^{\varepsilon}\left(u_{i}^{\varepsilon}\right)$. Combining the results of the steps 3 and 4 yields the convergence of $\tau^{\varepsilon}$ in $\mathbf{D}\left([0, \infty), L^{2}(-N, N)\right)$. Besides the finite-dimensional distributions converge to the distributions of continuous processes, so the weak limit of $\tau^{\varepsilon}$ belongs to $\mathbf{C}^{0}\left([0, \infty), L^{2}(-N, N)\right)$. As a consequence the convergence of $\tau^{\varepsilon}$ holds in $\mathbf{C}^{0}\left([0, \infty), L^{2}(-N, N)\right)$. It follows from the expression (3.6) of the time delay that $T^{\varepsilon}\left(u_{i}^{\varepsilon}\right)$ weakly converges in $\mathbf{C}^{0}([0, \infty), \mathbb{R})$.

Step 5. VARIAnCE of the limit process. We aim now at calculating the asymptotic variance. We still focus on the non trivial case $a=2$. First 
some calculations yield that:

$$
\lim _{L \rightarrow \infty} \frac{1}{L} \ln \mathbb{E}\left[\tau_{1}(L) \tau_{2}(L)\right]=\beta\left(k_{c}, h\right),
$$

where $\beta\left(k_{c}, h\right)=\alpha_{c}\left(k_{c}\right) k_{c}^{2}\langle\cos \phi\rangle_{m_{h}}$ and $\langle\cos \phi\rangle_{m_{h}}$ is given by (3.14). Since $\beta\left(k_{c}, h\right)$ is maximal when $h=0$ and $\left.\frac{\partial \beta}{\partial h}\left(k_{c}, h\right)\right|_{h=0^{+}}=-2 \pi,\left.\frac{\partial \beta}{\partial h}\left(k_{c}, h\right)\right|_{h=0^{-}}=$ $+2 \pi$, we have for any continuous function $\phi$ with compact support:

$$
\int_{-\infty}^{+\infty} \mathbb{E}\left[\tau_{1} \tau_{2}\right] \phi(h) d h=\frac{8 \phi(0)}{\pi \alpha_{c}\left(k_{c}\right) 2 k_{c}^{4} L} e^{\beta\left(k_{c}, 0\right) L}+\underset{L \rightarrow \infty}{o}\left(\frac{1}{L} e^{\beta\left(k_{c}, 0\right) L}\right) .
$$

Then it is easy to complete the proof of (3.12).

\section{Transmission through a ONE-DIMENSIONAL WEAKLY NONLINEAR RANDOM MEDIUM}

We consider a one-dimensional Helmholtz equation with a small random perturbation and study the problem of the decay of the transmission coefficient for large lengths with fixed output. It is well known that in the linear case we have exponential localization (Carmona (1985)), while with a strong nonlinear term we have a polynomial behavior of the transmittivity (Knapp et al. (1989)). Our main aim is to show that we still have an exponential decay of the transmission coefficient with a weak nonlinear term for fixed output.

We study the propagation of monochromatic waves through a finite, nonlinear, disordered slab $[0, L]$. The time harmonic scalar field $U$ satisfies the equation:

$$
U_{x x}+k_{0}^{2} n^{\varepsilon 2}\left(x,|U|^{2}\right) U=0,
$$

where $k_{0}$ is the vacuum wavenumber, and $n\left(x,|U|^{2}\right)$ is the index of refraction of the medium.

Outside the slab $[0, L]$, space is free and the index of refraction is equal to one. The model for the medium inside the slab is deduced from some optical media, whose indices of refraction are affected by the intensity of light and by random inhomogeneities. Therefore we shall take the index of refraction to have the form:

$$
n^{\varepsilon 2}\left(x,|U|^{2}\right)=n_{0}^{2}\left(1+m^{\varepsilon}(x)+\varepsilon^{a} \tilde{\gamma}|U|^{2}\right), \quad m^{\varepsilon}(x)=\varepsilon m\left(\frac{x}{\varepsilon^{2}}\right),
$$

where $m$ is an ergodic Markov process which describes the linear random perturbations of the index of refraction, $\tilde{\gamma}$ and $a$ are positive real constants. The small parameter $\varepsilon$ establishes ratios between the different scales. The employed scalings correspond to standard situations in optics (see Knapp et al. (1989)). We assume that the correlation radius of the perturbations and the wavelength are of the same order and are small compared to the size of the slab $L$. More exactly the correlation length and the wavelength are assumed to be of order $\varepsilon^{2}$ while $L$ is of order one. We therefore consider that the homogeneous wavenumber is $k^{\varepsilon}=k / \varepsilon^{2}$. We assume also that the amplitude of the random perturbation is weak, of order $\varepsilon$. Knapp et al. (1989) studied the decay of the transmission coefficient in the limit corresponding to these scalings when the size of the nonlinearity is of order one, i.e. the case $a=0$. In 1991 the same authors considered the case $a=2$, 
where only a slight modification of the arguments that are used in the linear case is needed. We shall consider a nonlinearity of order $\varepsilon^{a}$ for some real $a>0$. Of course the most relevant case is a small $a$.

We assume that a plane wave of amplitude $U_{0}$ is incoming from the right, so that there are an incident wave from the right and a reflected wave for $x \geq L$, and a transmitted wave for $x \leq 0$. Therefore the wave $U$ may be presented in the following form, outside the slab $[0, L]$ :

$$
\begin{cases}U(x)=U_{0}\left(e^{-i k^{\varepsilon}(x-L)}+R e^{i k^{\varepsilon}(x-L)}\right), & x \geq L, \\ U(x)=U_{0} T e^{-i k^{\varepsilon} x}, & x \leq 0\end{cases}
$$

where $R$ and $T$ are respectively the reflection and transmission coefficients. They depend on the length of the slab $L$ and on the amplitude of the incoming wave $U_{0}$ because of nonlinearity.

REMARK 4.1. We could have considered the nonlinear stationary Schrödinger equation with a random potential:

$$
-\psi_{x x}+V(x) \psi-\alpha|\psi|^{2} \psi=k^{2} \psi
$$

However the scalings we have just described have to be adapted.

We aim at analyzing the scattering problem which consists of the equation (4.1) with the boundary conditions (4.3). We introduce the normalized field $u$ such that $U(x)=U_{0} u(x)$. If we introduce the intensity of the incoming wave $w=\left|U_{0}\right|^{2}$, then the equation (4.1) can be rewritten as:

$$
u_{x x}+k^{\varepsilon^{2}}\left(1+m^{\varepsilon}(x)+\varepsilon^{a} \tilde{\gamma} w|u|^{2}\right) u=0 .
$$

The term $\varepsilon^{a} \tilde{\gamma} w$ governs the strength of the nonlinearity of the system. The boundary value problem $(4.5,4.3)$ can be replaced by an initial value problem parametrized by the output intensity $w_{0}=\left|T^{\varepsilon}\right|^{2} w$. Let us define the $\mathbb{R}$-valued functions $q^{\varepsilon}$ and $\theta^{\varepsilon}$ by $u(x)=\left|T^{\varepsilon}\right| q^{\varepsilon}(x) e^{-i \theta^{\varepsilon}(x)}$. Injecting this expression into (4.5), separating the real and imaginary multiples of $e^{-i \theta^{\varepsilon}}$, we find that $\theta_{x}^{\varepsilon}=k^{\varepsilon} / q^{\varepsilon 2}$ and $q^{\varepsilon}$ satisfies:

$$
\begin{cases}q_{x}^{\varepsilon}=p^{\varepsilon} & q^{\varepsilon}(0)=1 \\ p_{x}^{\varepsilon}=k^{\varepsilon 2}\left(q^{\varepsilon-3}-\left(1+m^{\varepsilon}(x)\right) q^{\varepsilon}-\varepsilon^{a} \gamma q^{\varepsilon 3}\right), & p^{\varepsilon}(0)=0\end{cases}
$$

where $\gamma=\tilde{\gamma} w_{0}$. The initial conditions of (4.6) are imposed by the boundary condition at $x=0$ in (4.3). Using the boundary condition at $x=L$, we get that the square modulus of the transmission coefficient $T^{\varepsilon}$ can be expressed as:

$$
\left|T^{\varepsilon}\right|^{2}(L)=\frac{2 k^{\varepsilon 2}}{E^{\varepsilon}(L)+k^{\varepsilon^{2}}-\frac{1}{4} k^{\varepsilon^{2}} \gamma \varepsilon^{a} q^{\varepsilon 4}(L)},
$$

where $E^{\varepsilon}$ is the energy given by:

$$
E^{\varepsilon}(L)=\frac{1}{2} p^{\varepsilon 2}(L)+V^{\varepsilon}\left(q^{\varepsilon}(L)\right), \quad V^{\varepsilon}(q)=\frac{k^{\varepsilon 2}}{2}\left(q^{2}+\frac{1}{q^{2}}+\frac{1}{2} \varepsilon^{a} \gamma q^{4}\right) .
$$

In order to explicit the periodic structure of the fast varying components of the processes $q^{\varepsilon}, p^{\varepsilon}$ and $E^{\varepsilon}$, we introduce the action angle variables (see 
Knapp et al. (1989)). The action $I$ is defined as a function of the energy $E$ by

$$
I^{\varepsilon}(E)=\frac{1}{2 \pi} \oint p d q^{\prime}=\frac{1}{2 \pi} \oint \sqrt{2 E-2 V^{\varepsilon}\left(q^{\prime}\right)} d q^{\prime} .
$$

Indeed, if you fix the energy at some value $E$, then the motion described by (4.8) is periodic, with a period given by $\pi^{\varepsilon}(E)=\oint \frac{d q^{\prime}}{p}=\oint \frac{d q^{\prime}}{\sqrt{2 E-2 V^{\varepsilon}\left(q^{\prime}\right)}}$ or else by $2 \pi \frac{\partial I^{\varepsilon}}{\partial E}$. The angle $\phi$ is defined as a function of $E$ and $q$ by

$$
\phi^{\varepsilon}(E, q)=-\int^{q} \frac{\partial p}{\partial I} d q^{\prime}=-\frac{2 \pi}{\pi^{\varepsilon}(E)} \int^{q} \frac{d q^{\prime}}{\sqrt{2 E-2 V^{\varepsilon}\left(q^{\prime}\right)}} .
$$

The transformation $(E, q) \rightarrow(I, \phi)$ can be inverted to give the functions $E^{\varepsilon}(I)$ and $Q^{\varepsilon}(I, \phi)$. Now we define $t$ by $x=t / \varepsilon^{2}$. In the scaled variable $t$, the correlation radius of the perturbation and the wavelength are of order 1 , while the size of the slab is of order $\varepsilon^{-2}$. So we study the random processes $I^{\varepsilon}\left(t / \varepsilon^{2}\right)$ and $\phi^{\varepsilon}\left(t / \varepsilon^{2}\right)$ in the scaled variable $t$; they are solutions of the differential equations:

$$
\left\{\begin{aligned}
\frac{d I^{\varepsilon}}{d t}\left(\frac{t}{\varepsilon^{2}}\right) & =\frac{1}{\varepsilon} m\left(\frac{t}{\varepsilon^{2}}\right) k^{2} h_{\phi}^{\varepsilon}\left(I^{\varepsilon}, \phi^{\varepsilon}\right), \\
\frac{d \phi^{\varepsilon}}{d t}\left(\frac{t}{\varepsilon^{2}}\right) & =-\frac{1}{\varepsilon^{2}} \omega^{\varepsilon}\left(I^{\varepsilon}\right)-\frac{1}{\varepsilon} m\left(\frac{t}{\varepsilon^{2}}\right) k^{2} h_{I}^{\varepsilon}\left(I^{\varepsilon}, \phi^{\varepsilon}\right),
\end{aligned}\right.
$$

where $h^{\varepsilon}(I, \phi)=(1 / 2) Q^{\varepsilon 2}(I, \phi)$ and $\omega^{\varepsilon}(I)=2 \pi / \pi^{\varepsilon}\left(E^{\varepsilon}(I)\right)$ are smooth functions and $h^{\varepsilon}$ is periodic with respect to $\phi$.

Lemma 4.2. For any integer $N$, we can expand $\omega^{\varepsilon}$ and $h^{\varepsilon}$

$$
\begin{gathered}
\omega^{\varepsilon}(I)=\sum_{j=0}^{N} a_{j}(I) \varepsilon^{j a}+\varepsilon^{(N+1) a} r^{\varepsilon}(I), \\
h^{\varepsilon}(I, \phi)=\sum_{j=0}^{N} H_{j}(I, \phi) \varepsilon^{j a}+\varepsilon^{(N+1) a} R^{\varepsilon}(I, \phi),
\end{gathered}
$$

where $a_{j}$ and $H_{j}$ are smooth functions, $r^{\varepsilon}$ and $R^{\varepsilon}$ are smooth functions uniformly bounded with respect to $I \in K, \phi \in \mathbb{R}$ and $\varepsilon \in(0,1]$ for any compact subset $K$ of $\mathbb{R}$. The explicit forms of the first terms $a_{0}$ and $H_{0}$ are:

$$
a_{0}(I)=2 k, \quad H_{0}(I, \phi)=\frac{1}{2}+\frac{I}{k}+\sqrt{\frac{I}{k}+\left(\frac{I}{k}\right)^{2}} \cos \phi .
$$

Proof. We first look for explicit representations of the functions $\omega^{\varepsilon}$ and $h^{\varepsilon}$. Let us fix $E$ and denote by $e_{3}^{\varepsilon}<e_{2}^{\varepsilon}<e_{1}^{\varepsilon}$ the roots of the polynomial $P_{E}^{\varepsilon}(z)=$ $-\frac{k^{2}}{2} \varepsilon^{a} \gamma z^{3}-k^{2} z^{2}+2 E z-k^{2}$. A straightforward study of $P_{E}^{\varepsilon}$ yields that $e_{3}^{\varepsilon}<$ $0<e_{2}^{\varepsilon}<1<e_{1}^{\varepsilon}$. As a consequence, the roots of $2\left(E-V^{\varepsilon}(q)\right)=P_{E}^{\varepsilon}\left(q^{2}\right) / q^{2}$ are $\sqrt{e_{2}^{\varepsilon}}$ and $\sqrt{e_{1}^{\varepsilon}}$. Then it appears that the bounds of the periodic motion described by (4.8) are $\sqrt{e_{2}^{\varepsilon}}$ and $\sqrt{e_{1}^{\varepsilon}}$. From the definition (4.9) of $I^{\varepsilon}$, it follows that:

$$
\frac{d I^{\varepsilon}}{d E}(E)=\frac{1}{\pi} \int_{\sqrt{\epsilon_{2}^{\varepsilon}(E)}}^{\sqrt{e_{1}^{\varepsilon}(E)}} \frac{q^{\prime} d q^{\prime}}{\sqrt{P_{E}^{\varepsilon}\left(q^{2}\right)}}
$$


We can recognize an elliptic integral, which can be rewritten (Abramowitz et al. (1965)) as:

$$
\frac{d I^{\varepsilon}}{d E}(E)=\frac{K\left(\rho^{\varepsilon}(E)\right)}{\pi}\left(\frac{\gamma \varepsilon^{a}}{2} k^{2}\left(e_{1}^{\varepsilon}(E)-e_{3}^{\varepsilon}(E)\right)\right)^{-1 / 2}
$$

where

$$
\rho^{\varepsilon}(I)=\frac{\epsilon_{1}^{\varepsilon}-\epsilon_{2}^{\varepsilon}}{e_{1}^{\varepsilon}-\epsilon_{3}^{\varepsilon}}\left(E^{\varepsilon}(I)\right),
$$

and $K$ is the complete elliptic integral:

$$
K(\rho)=\int_{0}^{\pi / 2}\left(1-\rho \sin ^{2} s\right)^{-1 / 2} d s .
$$

On the other hand, $\omega^{\varepsilon}(I)$ is equal to $\frac{d E^{\varepsilon}}{d I}(I)$, i.e. the inverse expression of (4.15). Since $E^{\varepsilon}(0)=k^{2}+\frac{k^{2}}{4} \gamma \varepsilon^{a}$ (indeed, if $I=0$, then $e_{1}^{\varepsilon}=e_{2}^{\varepsilon}=1$ and $\left.P_{E^{\varepsilon}(0)}^{\varepsilon}(1)=0\right)$, we get an explicit representation of $E^{\varepsilon}$ in terms of the roots $e_{i}^{\varepsilon}$ by integrating $\omega^{\varepsilon}(I)$. Finally we can recognize in the expression (4.10) of $\phi^{\varepsilon}$ an elliptic function of the third kind. Using the formulas given in Chapter 16, Abramowitz et al. (1965), the expression of $h^{\varepsilon}$ may be explicited by means of Jacobian elliptic functions:

$$
h^{\varepsilon}(I, \phi)=\frac{1}{2}\left(e_{1}^{\varepsilon}\left(E^{\varepsilon}(I)\right)-\left(e_{1}^{\varepsilon}-e_{2}^{\varepsilon}\right)\left(E^{\varepsilon}(I)\right) \operatorname{sn}^{2}\left(\frac{K\left(\rho^{\varepsilon}(I)\right)}{\pi} \phi, \rho^{\varepsilon}(I)\right)\right),
$$

where sn is the Jacobian sinus given by:

$$
S=\operatorname{sn}(\lambda, r) \leftrightarrow S=\sin \xi, \lambda=\int_{0}^{\xi} \frac{d s}{\sqrt{1-r \sin ^{2} s}} .
$$

In order to get the expansions of $\omega^{\varepsilon}$ and $h^{\varepsilon}$, we adopt the following strategy. We first expand the roots $e_{i}^{\varepsilon}$ in powers of $\varepsilon^{a}$. Then we expand the functions $\frac{d I^{\varepsilon}}{d E}(E), \omega^{\varepsilon}(I), E^{\varepsilon}(I)$ and $Q^{\varepsilon}(I, \phi)$ successively.

Let us first fix $E$. Then the roots $e_{1}^{\varepsilon}, e_{2}^{\varepsilon}$ and $e_{3}^{\varepsilon}$ of the polynomial $P_{E}^{\varepsilon}$ can be expanded in powers of $\varepsilon^{a}$. In particular the first order terms of the expansions are:

$$
\left\{\begin{aligned}
e_{1}^{\varepsilon} & =\frac{1}{k^{2}}\left(E+\sqrt{E^{2}-k^{4}}\right)+O\left(\varepsilon^{a}\right), \\
e_{2}^{\varepsilon} & =\frac{1}{k^{2}}\left(E-\sqrt{E^{2}-k^{4}}\right)+O\left(\varepsilon^{a}\right), \\
\epsilon_{3}^{\varepsilon} & =-\frac{2}{\gamma \varepsilon^{a}}+O(1), \\
\rho^{\varepsilon} & =\frac{1}{k^{2}} \sqrt{E^{2}-k^{4}} \gamma \varepsilon^{a}+O\left(\varepsilon^{2 a}\right) .
\end{aligned}\right.
$$

The notation $O\left(\varepsilon^{a}\right)$ is a shorthand for an expression of the form: $f_{1}(E) \varepsilon^{a}+$ $f_{2}(E) \varepsilon^{2 a}+\ldots$ where $f_{1}, f_{2}, \ldots$ are smooth deterministic functions.

The function $E \mapsto I^{\varepsilon}(E)$ is defined by (4.9) and its derivative by (4.16). It admits therefore an asymptotic expansion, whose first order term is:

$$
\frac{d I^{\varepsilon}}{d E}(E)=\frac{1}{2 k}+O\left(\varepsilon^{a}\right) .
$$


By inverting we get the expansion (4.12) of $\omega^{\varepsilon}$. By integrating with respect to $I$, we find that $E^{\varepsilon}(I)=E^{\varepsilon}(0)+2 k I+O\left(\varepsilon^{a}\right)$. Since $E^{\varepsilon}(0)=k^{2}+O\left(\varepsilon^{a}\right)$, we get:

$$
E^{\varepsilon}(I)=k^{2}\left(1+\frac{2 I}{k}\right)+O\left(\varepsilon^{a}\right) .
$$

The composition of the functions $I \mapsto E^{\varepsilon}(I)$ and $E \mapsto e_{j}^{\varepsilon}(E)$ therefore admits an expansion in powers of $\varepsilon^{a}$ :

$$
\left\{\begin{array}{l}
e_{1}^{\varepsilon}\left(E^{\varepsilon}(I)\right)=1+\frac{2 I}{k}+2 \sqrt{\frac{I}{k}+\left(\frac{I}{k}\right)^{2}}+O\left(\varepsilon^{a}\right), \\
e_{2}^{\varepsilon}\left(E^{\varepsilon}(I)\right)=1+\frac{2 I}{k}-2 \sqrt{\frac{I}{k}+\left(\frac{I}{k}\right)^{2}}+O\left(\varepsilon^{a}\right), \\
e_{3}^{\varepsilon}\left(E^{\varepsilon}(I)\right)=-\frac{2}{\gamma \varepsilon^{a}}+O(1), \\
\rho^{\varepsilon}\left(E^{\varepsilon}(I)\right)=2 \sqrt{\frac{I}{k}+\left(\frac{I}{k}\right)^{2}} \gamma \varepsilon^{a}+O\left(\varepsilon^{2 a}\right) .
\end{array}\right.
$$

Finally, from the representation (4.19) of $h^{\varepsilon}$ and the asymptotic expansions of the Jacobian functions (Abramowitz et al. (1965)), we can establish the expansion (4.13) of $h^{\varepsilon}$.

From now on, we fix an integer $N$ such that $(1+N) a>2$. We will see that the expansions of $\omega^{\varepsilon}$ and $h^{\varepsilon}$ at this order are sufficient for our purpose. We aim at studying the differential equations (4.11) satisfied by the process $\left(I^{\varepsilon}\left(t / \varepsilon^{2}\right), \phi^{\varepsilon}\left(t / \varepsilon^{2}\right)\right)_{t>0}$. Since the $\phi$-equation in (4.11) has a fast varying part $\omega^{\varepsilon}(I)$, we introduce new variables $\tau_{j}^{\varepsilon}(t), j=0, \ldots, N$ and a new random process $\psi^{\varepsilon}(t)$ defined by:

$$
\left\{\begin{array}{l}
\frac{d \tau_{j}^{\varepsilon}}{d t}(t)=-\varepsilon^{j a-2} a_{j}\left(I^{\varepsilon}\left(\frac{t}{\varepsilon^{2}}\right)\right), \quad \tau_{j}^{\varepsilon}(0)=0, \\
\phi^{\varepsilon}\left(\frac{t}{\varepsilon^{2}}\right)=\psi^{\varepsilon}(t)+\sum_{j=0}^{N} \tau_{j}^{\varepsilon}(t) .
\end{array}\right.
$$

$\tau_{0}^{\varepsilon}(t)$ is the fast varying component of the angle $\phi^{\varepsilon}\left(t / \varepsilon^{2}\right)$. Its behavior has a characteristic time of order $\varepsilon^{2}$ while the semi-fast varying components $\tau_{j}^{\varepsilon}(t), j=1, \ldots, N$ have characteristic times of order $\varepsilon^{2-j a}$ respectively. We can express the differential equations (4.11) with the scaled length $t$ and these new variables:

$$
\left\{\begin{aligned}
& \frac{d I^{\varepsilon}}{d t}= \sum_{j=0}^{N} \frac{1}{\varepsilon^{1-j a}} k^{2} m\left(\frac{t}{\varepsilon^{2}}\right) H_{j_{\phi}}\left(I^{\varepsilon}, \psi^{\varepsilon}+\sum_{l=0}^{N} \tau_{l}^{\varepsilon}\right) \\
&+\varepsilon^{(N+1) a-1} k^{2} m\left(\frac{t}{\varepsilon^{2}}\right) R_{\phi}^{\varepsilon}\left(I^{\varepsilon}, \psi^{\varepsilon}+\sum_{l=0}^{N} \tau_{l}^{\varepsilon}\right) \\
& \frac{d \psi^{\varepsilon}}{d t}=-\sum_{j=0}^{N} \frac{1}{\varepsilon^{1-j a}} k^{2} m\left(\frac{t}{\varepsilon^{2}}\right) H_{j I}\left(I^{\varepsilon}, \psi^{\varepsilon}+\sum_{l=0}^{N} \tau_{l}^{\varepsilon}\right) \\
&+\varepsilon^{(N+1) a-1} k^{2} m\left(\frac{t}{\varepsilon^{2}}\right) R_{I}^{\varepsilon}\left(I^{\varepsilon}, \psi^{\varepsilon}+\sum_{l=0}^{N} \tau_{l}^{\varepsilon}\right)-\varepsilon^{(N+1) a-2} r^{\varepsilon}\left(I^{\varepsilon}\right),
\end{aligned}\right.
$$


where $H_{0}$ is given by (4.14). In particular, $H_{0}$ is periodic with respect to its second variable. Moreover we can notice that the last term will be asymptotically negligible since $(N+1) a-2>0$. In fact, that is the motive for our choice of the integer $N$. By applying Theorem 6.1 in the appendix, we get that $\left(I^{\varepsilon}\left(t / \varepsilon^{2}\right)\right)_{t \geq 0}$ converges weakly to a Markov process $(I(t))_{t \geq 0}$ whose generator is given by:

$$
\mathcal{L}_{I}=\frac{\alpha_{c}(k) k^{4}}{2} \frac{\partial}{\partial I}\left(\frac{I}{k}+\left(\frac{I}{k}\right)^{2}\right) \frac{\partial}{\partial I}
$$

where $\alpha_{c}(k)$ has been defined by (2.8). Coming back to the square modulus of the transmission coefficient, we find that $\left(\left|T^{\varepsilon}\right|^{2}\left(L / \varepsilon^{2}\right)\right)_{L>0}$ converges weakly to the process $\mathcal{T}(L)=1 /(1+I / k(L))$. $(\mathcal{T}(L))_{L \geq 0}$ is a Markov process with generator $\mathcal{L}_{\mathcal{T}}$ given by:

$$
\mathcal{L}_{\mathcal{T}}=\frac{1}{2} \alpha_{c}(k) k^{2} \mathcal{T}^{2}\left(-\frac{\partial}{\partial \mathcal{T}}+(1-\mathcal{T}) \frac{\partial^{2}}{\partial \mathcal{T}^{2}}\right) .
$$

It is the same generator as in the linear case. Asymptotically, the square modulus of the transmission coefficient has the same law in the linear case and in the weak nonlinear case for the output problem. In particular,

$$
\lim _{L \rightarrow \infty} \frac{1}{L} \ln \mathbb{E}[\mathcal{T}(L)]=-\frac{\alpha_{c}(k) k^{2}}{8} .
$$

\section{Phase Difference}

We are now interested in the phase difference of the reflected wave in the situation described in Section 4. We consider the one-dimensional Helmholtz equation (4.1) where the index of refraction $n^{\varepsilon}$ is described by (4.2). We assume that a monochromatic plane wave with amplitude $U_{0}$ is incoming from the right. If we consider a free boundary condition at $x=0$, then the incident wave is scattered into a reflected wave and a transmitted wave, so that the wave has the form $U(x)=U_{0}\left(e^{-i k^{\varepsilon}(x-L)}+R^{\varepsilon} e^{i k^{\varepsilon}(x-L)}\right)$ for $x>$ $L$. From the previous section, we know that the modulus of the reflection coefficient is exponentially close to 1 for large lengths $L$. As a consequence, if we focus on the phase then it is convenient to analyze the problem with a totally reflecting condition at the interface $x=0$, so that $R^{\varepsilon}=e^{i \psi^{\varepsilon}}$. $\psi^{\varepsilon}$ is the phase difference of the reflected wave, that means the difference between the phase angle of the reflected phase and the phase angle of the incident wave at the interface $x=L$. If the medium is homogeneous inside the slab $[0, L]$ (i.e. $n \equiv n_{0}$ ), then $\psi^{\varepsilon}=2 k^{\varepsilon} L+\pi$. So the relative phase difference due to the fluctuations of the index of refraction is $\psi^{\varepsilon}-2 k^{\varepsilon} L-\pi$. We aim at describing $\psi^{\varepsilon}$ in the limit case described in Section 4 , where the fluctuation of the linear component of the index of refraction is of the form $m^{\varepsilon}(x)=\varepsilon m\left(x / \varepsilon^{2}\right)$ and the homogeneous wavenumber is $k^{\varepsilon}=k / \varepsilon^{2}$. We can now state the main result of this section.

Proposition 5.1. The phase difference $\psi^{\varepsilon}$ has the following structure when $\varepsilon \rightarrow 0$ :

$$
\psi^{\varepsilon}(L)=\frac{2 k L}{\varepsilon^{2}}+\frac{3 \gamma k L}{\varepsilon^{2-a}}+\phi^{\varepsilon}(L)+\pi
$$


where $\phi^{\varepsilon}$ converges weakly to a Brownian motion whose diffusive constant is $k^{2}\left(\alpha_{c}(k)+2 \alpha_{c}(0)\right)$, and $\gamma=\tilde{\gamma}\left|U_{0}\right|^{2}$.

Proof. By standard arguments (see Papanicolaou (1988)), we can find the equation which governs the evolution of the phase $\theta^{\varepsilon}(L):=\psi^{\varepsilon}(L)-2 k L / \varepsilon^{2}$ :

$\frac{d \theta^{\varepsilon}}{d L}=k\left(1+\cos \left(\frac{2 k L}{\varepsilon^{2}}+\theta^{\varepsilon}\right)\right)\left(\frac{1}{\varepsilon} m\left(\frac{L}{\varepsilon^{2}}\right)+\frac{2 \tilde{\gamma} w}{\varepsilon^{2-a}}\left(1+\cos \left(\frac{2 k L}{\varepsilon^{2}}+\theta^{\varepsilon}\right)\right)\right)$

If $a>2$, then it is obvious to check that the nonlinear term has no effect on the phase. If $a \in(0,2]$, then we introduce the semi-fast varying phase $\frac{3 \tilde{\gamma} w k L}{\varepsilon^{2}-a}$, so that

$$
\theta^{\varepsilon}(L)=\xi^{\varepsilon}(L)+\phi^{\varepsilon}(L)+\frac{3 \tilde{\gamma} w k L}{\varepsilon^{2-a}}+\pi,
$$

where $\xi^{\varepsilon}$ and $\phi^{\varepsilon}$ satisfy:

$$
\left\{\begin{aligned}
\frac{d \xi^{\varepsilon}}{d L} & =\frac{2 \gamma k}{\varepsilon^{2-a}}\left(\left(1-\cos \left(\frac{2 k L}{\varepsilon^{2}}+\frac{3 \tilde{\gamma} w k L}{\varepsilon^{2-a}}+\xi^{\varepsilon}(L)+\phi^{\varepsilon}(L)\right)\right)^{2}-\frac{3}{2}\right), \\
\frac{d \phi^{\varepsilon}}{d L} & =\frac{k}{\varepsilon} m\left(\frac{L}{\varepsilon^{2}}\right)\left(1-\cos \left(\frac{2 k L}{\varepsilon^{2}}+\frac{3 \tilde{\gamma} w k L}{\varepsilon^{2-a}}+\xi^{\varepsilon}(L)+\phi^{\varepsilon}(L)\right)\right) .
\end{aligned}\right.
$$

with the initial conditions $\xi^{\varepsilon}(0)=0$ and $\phi^{\varepsilon}(0)=0$. Then, applying Theorem 6.1 in the appendix, we get that the process $\left(\xi^{\varepsilon}(L), \phi^{\varepsilon}(L)\right)_{L>0}$ converges weakly to the Markov process $(0, \phi(L))_{L \geq 0}$ whose infinitesimal generator $\mathcal{L}_{\phi}$ is given by:

$$
\mathcal{L}_{\phi}=\frac{k^{2}}{2}\left(\alpha_{c}(k)+2 \alpha_{c}(0)\right) \frac{\partial^{2}}{\partial \phi^{2}}
$$

where $\alpha_{c}(k)$ has been defined by (2.8).

\section{Appendix : Multi-SCAlED DifFusion-APPROXimation}

In this appendix, we prove the multi-scaled diffusion-approximation theorems that are applied in this paper. General versions of these theorems can be found in Garnier (1996). We consider first the following problem: we aim at proving the weak convergence of the $\mathbb{R}^{d}$-valued process $X^{\varepsilon}$ defined by the system:

$$
\begin{aligned}
& \left\{\begin{aligned}
\frac{d X^{\varepsilon}}{d t}(t)= & \frac{1}{\varepsilon} F\left(X^{\varepsilon}(t), q^{\varepsilon}(t), h^{\varepsilon}(t), \tau_{1}^{\varepsilon}(t), \ldots, \tau_{N}^{\varepsilon}(t)\right) \\
& +\sum_{j=1}^{N} \frac{1}{\varepsilon^{1-j a}} G_{j}\left(X^{\varepsilon}(t), q^{\varepsilon}(t), h^{\varepsilon}(t), \tau_{1}^{\varepsilon}(t), \ldots, \tau_{N}^{\varepsilon}(t)\right) \\
& +\varepsilon^{b} R^{\varepsilon}\left(X^{\varepsilon}(t), q^{\varepsilon}(t), h^{\varepsilon}(t), \tau_{1}^{\varepsilon}(t), \ldots, \tau_{N}^{\varepsilon}(t)\right), \\
X^{\varepsilon}(0)= & x_{0} \in \mathbb{R}^{d} .
\end{aligned}\right. \\
& \begin{cases}\frac{d \tau_{j}^{\varepsilon}}{d t}(t)=\frac{1}{\varepsilon_{1}^{2-j a}} B_{j}\left(X^{\varepsilon}(t)\right) & , \tau_{j}^{\varepsilon}(0)=0, \quad j=1, \ldots, N, \\
\frac{d h^{\varepsilon}}{d t}(t)=\frac{1}{\varepsilon^{2}} A & , h^{\varepsilon}(0)=0 .\end{cases}
\end{aligned}
$$

In the following we shall denote $\tau=\left(\tau_{1}, \ldots, \tau_{N}\right)$ and $\nabla=\left(\frac{\partial}{\partial x_{j}}\right)_{j=1, \ldots, N}$.

We assume that:

- $a$ and $b$ are positive constants. 
- $A$ is a constant different from 0 and $B_{j}(x)$ are smooth functions for all $j=1, \ldots, N$.

- $q^{\varepsilon}(t)=q\left(t / \varepsilon^{2}\right)$ where $q$ is a Markov, stationary, ergodic process with generator $Q$, satisfying the Fredholm alternative.

- $F$ and $G_{j}$ are $\mathbb{R}^{d}$-valued functions which are periodic with respect to their third variables; they satisfy centering conditions: For any $x, \tau$, $\langle\mathbb{E}[F(x, q(0), h, \tau)]\rangle_{h}=0$ and $\left\langle\mathbb{E}\left[G_{j}(x, q(0), h, \tau)\right]\right\rangle_{h}=0$, where $\mathbb{E}[$.$] denotes$ the expectation with respect to the invariant probability measure of $q$ and $\langle.\rangle_{h}$ stands for an averaging over a period $T$ in $h$.

Instead of technical sharp conditions, we assume that $F$ and $G_{j}$ are smooth and have bounded partial derivatives in $x$.

- $R^{\varepsilon}$ is a function such that, for any compact subset $K$ of $\mathbb{R}^{d}$, we have:

$$
\sup _{\varepsilon \in(0,1]} \sup _{x \in K, q, h, \tau}\left|R^{\varepsilon}(x, q, h, \tau)\right|<\infty .
$$

- $N$ is an integer such that $(N+1) a>1$.

We introduce the infinitesimal generator $\mathcal{L}$ defined by:

$$
\mathcal{L} f(x)=\int_{0}^{\infty} d u\langle\mathbb{E}[F(x, q(0), h, \tau) . \nabla(F(x, q(u), h+A u, \tau) . \nabla f(x))]\rangle_{h}
$$

and claim that $\mathcal{L}$ does not depend on $\tau$.

THEOREM 6.1. The process $\left(X^{\varepsilon}(t)\right)_{t>0}$ given by $(6.1)$ converges weakly in $\mathrm{C}^{0}\left([0, \infty), \mathbb{R}^{d}\right)$ to the diffusion Markov process $X$ generated by $\mathcal{L}$ of $(6.4)$.

This theorem is actually a multi-scaled version of Theorem 2-7 Papanicolaou et al. (1976). We will use the perturbed test function method (Kushner (1984)) to prove it.

Proof. $\left(q^{\varepsilon}(t), h^{\varepsilon}(t), \tau^{\varepsilon}(t), X^{\varepsilon}(t)\right)_{t \geq 0}$ is a Markov process with generator $\mathcal{L}^{\varepsilon}$ :

$$
\begin{aligned}
\mathcal{L}^{\varepsilon}= & \frac{1}{\varepsilon^{2}}\left(Q+A \frac{\partial}{\partial h}\right)+\sum_{j=1}^{N} \frac{1}{\varepsilon^{2-j a}} B_{j}(x) \frac{\partial}{\partial \tau_{j}}+\frac{1}{\varepsilon} F(x, q, h, \tau) . \nabla \\
& +\sum_{j=1}^{N} \frac{1}{\varepsilon^{1-j a}} G_{j}(x, q, h, \tau) . \nabla+\varepsilon^{b} R^{\varepsilon}(x, q, h, \tau) . \nabla .
\end{aligned}
$$

According to Kushner (1984) (see Chapter III Theorems 2 and 4), the most important fact to establish is:

For any bounded, $\mathcal{C}^{\infty}$ function $f$, we can find a family of functions $f^{\varepsilon}$ such that, for any compact subset $K$ of $\mathbb{R}^{d}$ :

$$
\begin{array}{r}
\sup _{x \in K, q, h, \tau}\left|f^{\varepsilon}(x, q, h, \tau)-f(x)\right| \stackrel{\varepsilon \rightarrow 0}{\longrightarrow} 0, \\
\sup _{x \in K, q, h, \tau}\left|\mathcal{L}^{\varepsilon} f^{\varepsilon}(x, q, h, \tau)-\mathcal{L} f(x)\right| \stackrel{\varepsilon \rightarrow 0}{\longrightarrow} 0 .
\end{array}
$$

Let $f$ be a bounded, $\mathcal{C}^{\infty}$ function. We define $f^{\varepsilon}$ by:

$$
f^{\varepsilon}(x, q, h, \tau)=f(x)+\sum_{j=0}^{N} \varepsilon^{1+j a} f_{1+j}(x, q, h, \tau)+\varepsilon^{2} f_{N+2}(x, q, h, \tau) .
$$


$f^{\varepsilon}$ is a correction of $f$, whose corrective terms $f_{j}$ will be chosen such that (6.6) and $(6.7)$ will be satisfied. If we apply the generator $\mathcal{L}^{\varepsilon}$ to $f^{\varepsilon}$ then we find:

$$
\begin{aligned}
& \mathcal{L}^{\varepsilon} f^{\varepsilon}=\frac{1}{\varepsilon}\left(\left(Q+A \frac{\partial}{\partial h}\right) f_{1}+F . \nabla f\right) \\
& +\sum_{j=1}^{N} \frac{1}{\varepsilon^{1-j a}}\left(\sum_{i=1}^{j} B_{i} \frac{\partial}{\partial \tau_{i}} f_{j+1-i}+G_{j} \cdot \nabla f+\left(Q+A \frac{\partial}{\partial h}\right) f_{j+1}\right) \\
& +\left(\left(Q+A \frac{\partial}{\partial h}\right) f_{N+2}+F . \nabla f_{1}\right)+O\left(\varepsilon^{a \wedge b \wedge((N+1) a-1)}\right) .
\end{aligned}
$$

Our first aim is to get $\mathcal{L}^{\varepsilon} f^{\varepsilon}$ without any divergent term. The second one will be to get an expression with a $O(1)$ term independent of $q, h, \tau$. Therefore we define the functions $f_{j}$ as follows:

$$
f_{1}(x, q, h, \tau)=-\left(Q+A \frac{\partial}{\partial h}\right)^{-1}(F(x, q, h, \tau) \cdot \nabla f(x)) .
$$

$\left(Q+A \frac{\partial}{\partial h}\right)$ does not have an inverse. However, denoting $\dot{h}=h \bmod T$, the Markov $\left(q^{\varepsilon}, h^{\varepsilon}\right)$ satisfies the Fredholm alternative. Therefore $\left(Q+A \frac{\partial}{\partial h}\right)$ has an inverse on the subspace of periodic functions which are centered under the invariant probability $\mathbb{P} \otimes \mathbb{1}_{[0, T)}$ of $(q, \dot{h})$. Thus the previous expression is well-defined since $\langle\mathbb{E}[F(x, q(0), h, \tau) . \nabla f(x)]\rangle_{h}=0$. An explicit expression of $f_{1}$ is:

$$
f_{1}(x, q, h, \tau)=\int_{0}^{\infty} d u \mathbb{E}_{q}[F(x, q(u), h+A u, \tau) . \nabla f(x)] .
$$

For $j=1, \ldots, N$, we define by induction $f_{j+1}$ :

$$
\begin{gathered}
f_{j+1}(x, q, h, \tau)=-\left(Q+A \frac{\partial}{\partial h}\right)^{-1} \\
\left(\sum_{i=1}^{j} B_{i}(x) \frac{\partial}{\partial \tau_{i}} f_{j+1-i}(x, q, h, \tau)+G_{j}(x, q, h, \tau) . \nabla f(x)\right) .
\end{gathered}
$$

The function between parentheses is centered. Indeed, on the one hand $G_{j}$ is centered by assumption, and on the other hand we can show that $f_{j}$ is centered by induction with respect to $j$, since the operators $\left(Q+A \frac{\partial}{\partial h}\right)^{-1}$ and $\frac{\partial}{\partial \tau_{i}}$ do not modify the centering.

Finally we adopt for $f_{N+2}$ the following expression:

$$
\begin{gathered}
f_{N+2}(x, q, h, \tau)=-\left(Q+A \frac{\partial}{\partial h}\right)^{-1} \\
\left(F(x, q, h, \tau) . \nabla f_{1}(x, q, h, \tau)-\left\langle\mathbb{E}\left[F(x, q(0), h, \tau) . \nabla f_{1}(x, q(0), h, \tau)\right]\right\rangle_{h}\right)
\end{gathered}
$$

which is well-defined because of the centering of the integrated term.

Adopting this definition for $f^{\varepsilon}$, we have:

$$
\mathcal{L}^{\varepsilon} f^{\varepsilon}(x, q, h, \tau)=\mathcal{L} f(x)+O\left(\varepsilon^{a \wedge b \wedge((N+1) a-1)}\right),
$$

where $\mathcal{L}$ is given by (6.4). Thus $f^{\varepsilon}$ satisfies (6.6) and (6.7). 
Finally, $G_{j}$ and the semi-fast varying phases $\tau_{j}^{\varepsilon}(t), j=1, \ldots, N$ disappear in the diffusion limit. They are negligible compared to $F$ and the fast varying phase $A t / \varepsilon^{2}$ respectively.

The above result deals with a diffusion-approximation problem with fast and semi-fast varying phases. We are now concerned in proving a diffusionapproximation result where an ultra-fast varying phase competes with a random process. This result is needed in Section 2. We consider the following problem: we aim at proving the weak convergence of the $\mathbb{R}^{d}$-valued process $X^{\varepsilon}$ defined by the system:

$$
\begin{cases}\frac{d X^{\varepsilon}}{d t}(t)=\frac{1}{\varepsilon^{\varepsilon}} F\left(X^{\varepsilon}(t), q^{\varepsilon}(t), \phi^{\varepsilon}(t)\right), & X^{\varepsilon}(0)=x_{0} \in \mathbb{R}^{d}, \\ \frac{d \phi^{\varepsilon}}{d t}(t)=\frac{1}{\varepsilon^{2+c}} C, & \phi^{\varepsilon}(0)=0 .\end{cases}
$$

We assume that:

- $c$ and $C$ are positive constants.

- $q^{\varepsilon}(t)=q\left(t / \varepsilon^{2}\right)$ where $q$ is a Markov, stationary, ergodic process with generator $Q$, satisfying the Fredholm alternative.

- $F$ is a smooth $\mathbb{R}^{d}$-valued function which is periodic with respect to its third variable and satisfies $\langle\mathbb{E}[F(x, q(0), \phi)]\rangle_{\phi}=0$, where $\mathbb{E}[$.$] denotes the$ expectation with respect to the invariant probability measure of $q$ and $\langle\cdot\rangle_{\phi}$ stands for an averaging over a period in $\phi$.

TheOREM 6.2. The process $\left(X^{\varepsilon}(t)\right)_{t>0}$ given by (6.8) converges weakly in $\mathrm{C}^{0}\left([0, \infty), \mathbb{R}^{d}\right)$ to the diffusion Markov process $X$ generated by the infinitesimal generator $\mathcal{L}$ given by:

$$
\mathcal{L} f(x)=\int_{0}^{\infty} d u \mathbb{E}\left[\langle F(x, q(0), .)\rangle_{\phi} \cdot \nabla\left(\langle F(x, q(u), .)\rangle_{\phi} . \nabla f(x)\right)\right] .
$$

An infinite-dimensional version of this theorem is also available in Bailly (1996).

Proof. $\left(q^{\varepsilon}(t), \phi^{\varepsilon}(t), X^{\varepsilon}(t)\right)_{t \geq 0}$ is a Markov process with generator $\mathcal{L}^{\varepsilon}$ given by:

$$
\mathcal{L}^{\varepsilon}=\frac{C}{\varepsilon^{2+c}} \frac{\partial}{\partial \phi}+\frac{1}{\varepsilon^{2}} Q+\frac{1}{\varepsilon} F(x, q, \phi) \cdot \nabla
$$

We still use the perturbed test function method to prove this theorem. We denote by $M$ an integer that satisfies $M c>1$. Let $f$ be a bounded, $\mathcal{C}^{\infty}$ function. We define $f^{\varepsilon}$ by:

$$
\begin{aligned}
f^{\varepsilon}(x, q, \phi)=f(x) \quad & +\varepsilon f_{1,0}(x, q)+\sum_{j=1}^{M} \varepsilon^{1+j c} f_{1, j}(x, q, \phi) \\
& +\varepsilon^{2} f_{2,0}(x, q)+\varepsilon^{2+c} f_{2,1}(x, q, \phi) .
\end{aligned}
$$


Applying the generator $\mathcal{L}^{\varepsilon}$ to $f^{\varepsilon}$, we get:

$$
\begin{aligned}
\mathcal{L}^{\varepsilon} f^{\varepsilon}= & \frac{1}{\varepsilon}\left(Q f_{1,0}(x, q)+F(x, q, \phi) \cdot \nabla f(x)+C \frac{\partial f_{1,1}}{\partial h}\right) \\
& +\sum_{j=1}^{M-1} \frac{1}{\varepsilon^{1-j c}}\left(C \frac{\partial f_{1, j+1}}{\partial \phi}(x, q, \phi)+Q f_{1, j}(x, q, \phi)\right) \\
& +\left(C \frac{\partial f_{2,1}}{\partial \phi}(x, q, \phi)+Q f_{2,0}(x, q)+F(x, q, \phi) \cdot \nabla f_{1,0}(x, q)\right) \\
& +O\left(\varepsilon^{c \wedge(M c-1)}\right) .
\end{aligned}
$$

Defining

$$
\begin{gathered}
f_{1,0}(x, q)=-Q^{-1}\langle F(x, q, .)\rangle_{\phi} \cdot \nabla f(x) \\
f_{1,1}(x, q, \phi)=-\frac{1}{C} \int_{0}^{\phi} d \phi^{\prime}\left(F\left(x, q, \phi^{\prime}\right)-\langle F(x, q, .)\rangle_{\phi}\right) \cdot \nabla f(x)+c_{1,1}(x, q) .
\end{gathered}
$$

the $O\left(\frac{1}{\varepsilon}\right)$ term in $\mathcal{L}^{\varepsilon} f^{\varepsilon}$ becomes 0 , whatever $c_{1,1}$ is. Choosing now $c_{1,1}$ as:

$$
c_{1,1}(x, q)=\left\langle\frac{1}{C} \int_{0} d \phi^{\prime}\left(F\left(x, q, \phi^{\prime}\right)-\langle F(x, q, .)\rangle_{\phi}\right) \cdot \nabla f(x)\right\rangle_{\phi},
$$

we get that $f_{1,1}$ is periodic with mean 0 . Defining by induction

$$
f_{1, j+1}(x, q, \phi)=-\frac{1}{C} \int_{0}^{\phi} d \phi^{\prime} Q f_{1, j}\left(x, q, \phi^{\prime}\right)+c_{1, j+1}(x, q),
$$

where

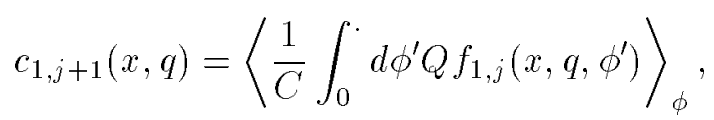

all the terms of order $\frac{1}{\varepsilon^{1-j c}}$ are equal to 0 . Finally, we take $f_{2,0}$ and $f_{2,1}$ to be

$$
\begin{gathered}
f_{2,0}(x, q)=-Q^{-1} \\
\left(\langle F(x, q, .)\rangle_{\phi} \cdot \nabla f_{1,0}(x, q)-\mathbb{E}\left[\langle F(x, q(0), .)\rangle_{\phi} \cdot \nabla f_{1,0}(x, q(0))\right]\right), \\
f_{2,1}(x, q, \phi)=-\frac{1}{C} \int_{0}^{\phi} d \phi^{\prime}\left(F\left(x, q, \phi^{\prime}\right)-\langle F(x, q, .)\rangle_{\phi}\right) \cdot \nabla f_{1,0}(x, q),
\end{gathered}
$$

which are well-defined because of the Fredholm alternative and of the centering conditions. As a consequence $f^{\varepsilon}$ satisfies the condition (6.6), and

$$
\mathcal{L}^{\varepsilon} f^{\varepsilon}(x, q, \phi)=\mathcal{L} f(x)+O\left(\varepsilon^{c \wedge(M c-1)}\right),
$$

where $\mathcal{L} f=\mathbb{E}\left[\langle F(x, q(0), .)\rangle_{\phi} \cdot \nabla f_{1,0}(x, q(0))\right]$ is given by $(6.9)$ so that $f^{\varepsilon}$ fulfills $(6.7)$.

REMARK 6.3. In this appendix we could have presented only one theorem by considering a more general situation where we would have taken into account all the terms that we dealt with successively in Theorems 6.1 and 6.2. However, for the sake of simplicity in the notations, we preferred to break our presentation into two parts.

\section{ACKNOWLEDGEMENTS}

It is a pleasure to acknowledge numerous and helpful discussions with J. P. Fouque on several of the topics discussed here above. 


\section{REFERENCES}

Abramowitz, M. and STEgun, I. (1965). Handbook of mathematical functions, Dover Publications, New-York.

Aldous, D. (1978). Stopping times and tightness. Ann. Prob. 6 335-340.

Bailly, F. (1996) Ph-D thesis. Paris-Sud.

Bouc, R. and PARDoux, E. (1984). Asymptotic analysis of P.D.E.'s with wide-band noise disturbances and expansions of the moments. Stochastic analysis and Appl. $2369-422$.

Carmona, R. (1985). The random Schrödinger equation, in: Ecole d'été de Probabilités de Saint-Flour, Hennequin, P. L., ed. Lecture Notes in Mathematics, Springer.

Carmona, R. and Lacroix, J. (1990). Spectral theory of random Schrödinger operators. Birkhauser.

Collins, R. E. (1960). Field theory of guided waves. Mac Graw-Hill, New York.

Desvillard, P. and Souillard, B. (1986). Polynomially decaying transmission for the nonlinear Schrödinger equation in a random medium. J. of Stat. Phys. 43 423439.

FARIS, W. G. and Tsay, W. J. (1994). Time delay in random scattering. SIAM J. Appl. Math. 54 443-455.

Garnier, J. (1996) Ph-D thesis. Ecole Polytechnique.

Grofll J. E. (1968). A circular harmonic computer analysis for rectangular dielectric waveguides. Bell. Syst. Tech. J. 48.

Jauch, J., Sinha, K. and Misra, B. (1972). Time delay in scattering processes. Helv. Phys. Acta 45 398-426.

Kesten, J. B. and Papanicolaou, G. (1979). A limit theorem for turbulent diffusion. Comm. Math. Phys. 65 97-128.

Knapp, R., Papanicolaou, G. and White, B. (1989). Nonlinearity and localization in one-dimensional random media, in: Disorder and Nonlinearity Bishop, A. R., Campbell, I. K. and Pnevmatikos, S., eds. Springer.

Knapp, R., Papanicolaou, G. and White, B. (1991). Transmission of waves by a nonlinear random medium. J. of Stat. Phys. 63 567-583.

KushNER, H. J. (1984). Approximation and weak convergence methods for random processes. MIT Press.

Metivier M. (1984). Convergence faible et principe d'invariance pour des martingales à valeurs dans des espaces de Sobolev. Rapport interne CMAP-Ecole Polytechnique. 106.

Papanicolaou, G. (1988). Waves in one-dimensional random media, in: Ecole d'été de Probabilités de Saint-Flour, Hennequin, P. L., ed. Lecture Notes in Mathematics, Springer.

Papanicolaou, G., Stroock, D. and Varadhan, S. R. S. (1976). Martingale approach to some limit theorems, in: Statistical Mechanics and Dynamical Systems Ruelle, D., ed. Duke Turbulence Conf. (Duke Univ. Math. Series III, Part VI).

CMap, Ecole Polytechnique, F-91128 Palaiseau Cedex, France. E-mail: garnier@paris . polytechnique.fr 